\title{
Quantitative input-output dynamics of a c-di-GMP signal-transduction cascade in Vibrio cholerae
}

Andrew A. Bridges ${ }^{1^{\star}}$, Jojo A. Prentice ${ }^{1^{\star}}$, Chenyi Fei ${ }^{1,2}$, Ned S. Wingreen ${ }^{1,2 \#}$, Bonnie L. Bassler ${ }^{1,3 \#}$

${ }^{*}$ These authors contributed equally

\#Correspondence to: wingreen@princeton.edu bbassler@princeton.edu

\author{
Affiliations: \\ ${ }^{1}$ Department of Molecular Biology, Princeton University, Princeton, New Jersey 08544, USA. \\ ${ }^{2}$ Lewis-Sigler Institute for Integrative Genomics, Princeton University, Princeton, New Jersey \\ 08544, USA. \\ ${ }^{3}$ The Howard Hughes Medical Institute, Chevy Chase, MD 20815, USA.
}

\section{Abstract}

Bacterial biofilms are multicellular communities that collectively overcome environmental

2 threats and clinical treatments. To regulate the biofilm lifecycle, bacteria commonly transduce

3 sensory information via the second-messenger molecule cyclic diguanylate (c-di-GMP). Using

4 experimental and modeling approaches, we quantitatively capture c-di-GMP signal transmission

5 via the bifunctional polyamine receptor NspS-MbaA, from ligand binding to output, in the pathogen

6 Vibrio cholerae. Upon binding of norspermidine or spermidine, NspS-MbaA synthesizes or

7 degrades c-di-GMP, respectively, which in turn, drives alterations specifically to biofilm gene

8 expression. A longstanding question is how output specificity is achieved via c-di-GMP, a

9 diffusible molecule that regulates dozens of effectors. We show that NspS-MbaA signals locally

10 to specific effectors, sensitizing $V$. cholerae to polyamines. However, local signaling is not

11 required for specificity, as changes to global cytoplasmic c-di-GMP levels can selectively regulate

12 biofilm genes. This work establishes the input-output dynamics underlying c-di-GMP signaling,

13 which could be useful for developing bacterial manipulation strategies. 


\section{Introduction}

Bacteria have the versatility to modify their lifestyles in response to shifting challenges.

15 Commonly, bacteria resist threats by forming multicellular communities called biofilms, in which

16 bacteria attach to surfaces and collectively produce an extracellular matrix (Flemming et al.,

17 2016). Advantages that accrue to biofilm-resident cells include resistance to antimicrobial

18 compounds, protection from predators, and the ability to collectively acquire nutrients (Flemming

19 and Wingender, 2010; Mah et al., 2003; Shaw et al., 2004). Biofilm bacteria can return to the

20 individual, free-swimming state by degrading the biofilm matrix and initiating motility, which

21 together facilitate escape from the biofilm and spread to new territories (Guilhen et al., 2017). The

22 ability to repeatedly transition between planktonic and biofilm states is central to the disease

23 process for many pathogenic bacteria, and such lifestyle flexibility confounds clinical treatment

24 (Guilhen et al., 2017). Interventions that manipulate biofilm formation and/or dispersal hold

25 promise as therapeutics to combat globally-important pathogenic bacteria (Trebino et al., 2021).

Biofilm formation and dispersal are controlled by environmental stimuli. Bacteria detect

27 many of these stimuli with membrane-bound receptors that transduce signals internally.

28 Information flow typically converges on a set of key intracellular regulators, commonly including

29 the bacterial second-messenger molecule cyclic diguanylate (c-di-GMP) (Jenal et al., 2017).

30 Across the bacterial domain, high levels of c-di-GMP are associated with surface attachment and

31 biofilm matrix production while low levels drive inhibition of biofilm formation and enhanced motility

32 (Römling et al., 2013). Receptors controlling c-di-GMP levels contain ligand binding domains

33 which regulate the activities of attached domains responsible for c-di-GMP synthesis and/or

34 degradation. c-di-GMP synthesis occurs via diguanylate cyclase domains, which harbor catalytic

35 "GGDEF" motifs. c-di-GMP is degraded by phosphodiesterase domains characterized by either

36 "EAL" or "HD-GYP" motifs (Dow et al., 2006; Simm et al., 2004). The outputs of these enzymes

37 are decoded by c-di-GMP-binding effectors that modulate transcription, translation, or protein 
activity, which in turn, control bacterial behaviors (Jenal et al., 2017). For example, in the global pathogen Vibrio cholerae, the model organism used in the present work, the VpsT and VpsR transcription factors bind to c-di-GMP and subsequently activate expression of vps genes encoding vibrio polysaccharide biosynthesis enzymes (Conner et al., 2017). The Vps machinery promotes biofilm formation by facilitating matrix production.

Bacterial genomes often encode dozens of c-di-GMP metabolizing receptors (Dahlstrom and O'Toole, 2017). V. cholerae possesses 31 diguanylate cyclases, 12 phosphodiesterases, and 10 proteins containing both diguanylate cyclase and phosphodiesterase motifs (Conner et al., 2017). This large array of receptors presumably allows V. cholerae to respond to many cues. However,

47 it is mysterious how the pertinent sensory information is integrated to accurately regulate downstream processes including biofilm formation and dispersal. In V. cholerae and most other studied species, the ligands regulating specific receptors that harbor c-di-GMP synthesis and/or degradation domains remain unidentified (Jenal et al., 2017). Thus, a quantitative understanding of the inputoutput dynamics underlying how c-di-GMP signaling modules convert ligand binding events into

52 alterations in phenotypes remains elusive. Furthermore, despite the diffusibility of the c-di-GMP

53 molecule, genetic evidence suggests that some c-di-GMP-metabolizing enzymes show specificity,

54 controlling only small subsets of c-di-GMP-binding effectors (Dahlstrom and O'Toole, 2017; Hengge,

55 2021). For example, deletion of genes specifying particular diguanylate cyclases reduces biofilm

56 formation while not altering motility, whereas deletion of others activates motility but does not change

57 biofilm formation (Zamorano-Sánchez et al., 2019). How specificity is achieved by c-di-GMP signaling

58 modules remains a topic of intense interest (Dahlstrom and O'Toole, 2017; Hengge, 2021).

61 (Bridges and Bassler, 2021). MbaA is a bifunctional enzyme, harboring both diguanylate cyclase

62 (GGDEF domain) and phosphodiesterase (EAL domain) activities (Fig. 1A). MbaA detects 
63 polyamine ligands via interaction with the periplasmic binding protein NspS (Karatan et al., 2005;

64 Sobe et al., 2017). NspS binds both norspermidine, a rare polyamine produced by Vibrionaceae 65 and select other organisms, and spermidine, a nearly ubiquitous polyamine that is not produced by V. cholerae (Fig. 1A) (Young et al., 2021). Norspermidine binding to NspS is thought to drive

67 NspS-MbaA association and this interaction favors MbaA diguanylate cyclase activity, promoting

68 the biofilm state (Cockerell et al., 2014). Conversely, when NspS is in the apo state or when it is

69 bound to spermidine, NspS does not associate with MbaA, and MbaA exhibits phosphodiesterase

70 activity, repressing biofilm formation and driving the planktonic state (Fig. 1A) (Cockerell et al.,

71 2014). Thus, NspS ligand occupancy dictates MbaA activity, which in turn, controls cytoplasmic

72 c-di-GMP levels and whether $V$. cholerae forms or exits from biofilms. Notably, because the

73 ligands for NspS-MbaA are known, and because MbaA can both produce and degrade c-di-GMP,

74 this system is uniquely configured for a case study that incorporates all steps in c-di-GMP signal

75 transduction - from ligand binding to phenotypic output.

To develop a quantitative understanding of signal transduction through the NspS-MbaA

77 circuit, we formulate a mathematical model describing NspS-MbaA signaling from ligand binding

78 to changes in c-di-GMP levels. Companion experiments show that NspS-MbaA detects its ligands

79 in the periplasm with sub-nanomolar affinity. This sensitivity enables NspS-MbaA to respond to

80 external polyamine fluctuations in the face of high affinity polyamine import by the cell. In turn, the

81 c-di-GMP produced or degraded by MbaA regulates biofilm gene expression with high specificity.

82 We address the long-standing issue of whether c-di-GMP signaling specificity is a consequence

83 of localized c-di-GMP transmission between specific pairs of receptors and effectors, or

84 alternatively, if changes in the global cytoplasmic c-di-GMP pool are detected only by particular 85 effectors based strictly on their relative affinities for c-di-GMP (Hengge, 2021). We find that MbaA 86 appears to channel c-di-GMP information exclusively through a localized mechanism at low ligand 87 concentrations, whereas at high ligand concentrations it alters the global cytoplasmic c-di-GMP 
pool. Notably, however, across the large range of tested polyamine concentrations, MbaA maintains signaling specificity, as among all c-di-GMP-responsive genes, changes only occur in the expression of those involved in biofilm formation. By assaying other receptors that produce c-

91 di-GMP, we show that localized c-di-GMP signaling is not a prerequisite for specific control of

92 biofilm gene expression in V. cholerae, as specificity can also be achieved through changes to

93 the global cytoplasmic c-di-GMP pool. Regarding the NspS-MbaA circuit, the consequence of

94 local signaling is increased sensitivity to polyamine ligands, endowing $V$. cholerae with the ability

95 to detect physiological polyamine concentrations. To our knowledge, the work presented here

96 provides the first quantitative study of c-di-GMP signal transmission from ligand binding to

97 changes in the behaviors of cell collectives.

\section{$\underline{\text { Results }}$}

\section{A mathematical model describes the experimentally observed NspS-MbaA input-output dynamics}

To quantitatively describe the relationship between polyamine signal input and c-di-GMP output via the NspS-MbaA circuit, we relied on our previous experimental measurements that exploited an established fluorescence-based live-cell c-di-GMP reporter (Bridges and Bassler, 2021; Zamorano-Sánchez et al., 2019; Zhou et al., 2016). In these assays, exogenous norspermidine and spermidine mixtures were supplied to cultures harboring a reporter whose

103 output tracks linearly with cytoplasmic c-di-GMP concentrations (Zhou et al., 2016). The

104 experimentally obtained wildtype c-di-GMP reporter results exhibit three regimes (Fig. 1B, left and 105 middle panels): 1) a basal level of c-di-GMP, which is maintained when up to $10 \mu \mathrm{M}$ exogenous 106 norspermidine and up to $1 \mu \mathrm{M}$ exogenous spermidine are added, 2) a reduced c-di-GMP level, 107 which is established when high $(\geq 10 \mu \mathrm{M})$ spermidine and low $(\leq 10 \mu \mathrm{M})$ norspermidine are 108 provided, and 3) an elevated c-di-GMP level, which is achieved when high $(\geq 50 \mu M)$ 109 norspermidine is supplied, irrespective of the amount of administered spermidine. In the $\Delta n s p S$ 110 strain (Fig. 1C, left and middle panels), basal c-di-GMP levels are lower than the wildtype basal 
111 level due to constitutive MbaA phosphodiesterase activity, and there is no response to exogenous

112 polyamine mixtures. The $\triangle m b a A$ strain (Fig. 1D, left and middle panels) is also incapable of

113 responding to exogenous polyamines; however, it harbors slightly higher c-di-GMP levels than

114 the untreated wildtype strain due to the lack of basal MbaA phosphodiesterase activity. The final

115 experimental dataset we employed for model construction was that of the $\Delta$ potD1 strain (Fig. 1E,

116 left and middle panels), which is incapable of norspermidine and spermidine import due to the

117 lack of a functional PotABCD1 ABC-family transporter (McGinnis et al., 2009). In the absence of

118 polyamine import, our previous results suggest that self-secreted norspermidine accumulates in

119 the periplasm, leading to high MbaA diguanylate cyclase activity (Fig. 1E, left panel). Therefore,

120 the $\Delta$ potD1 strain exhibits elevated basal c-di-GMP levels relative to the wildtype, no response to

121 exogenous norspermidine, and an attenuated response to exogenous spermidine (Fig. 1E, middle

122 panel). 


\section{Figure 1}

A

Norspermidine (Nspd)
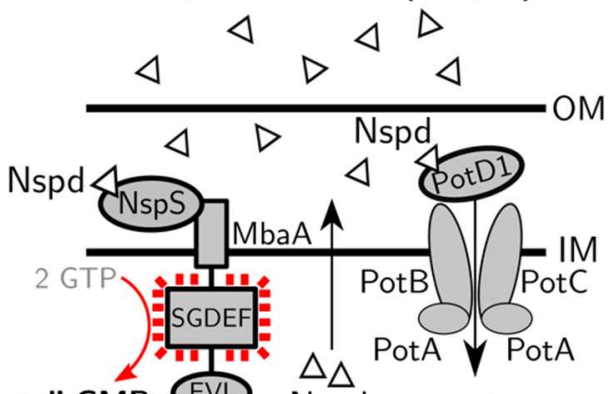

c-di-GMP EVD Nspd $\triangle \triangle$

B
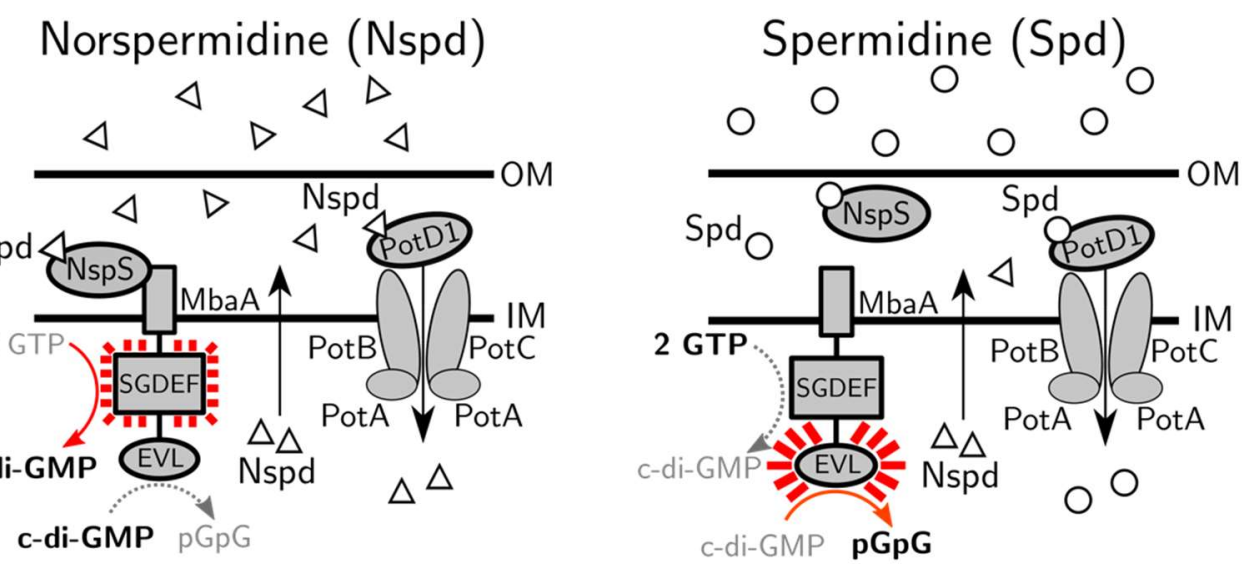

B

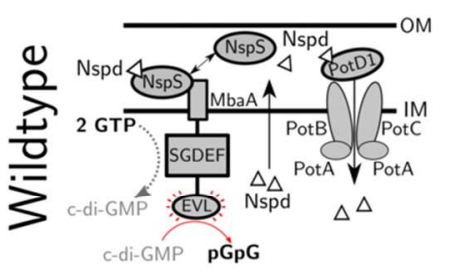

C

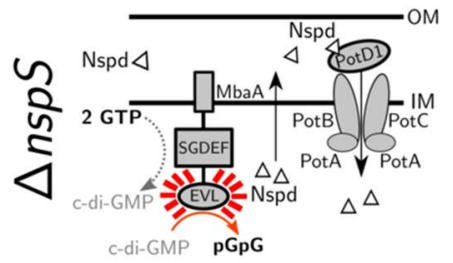

D

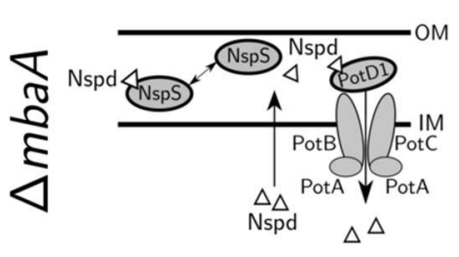

E

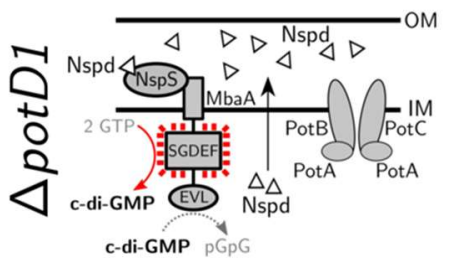

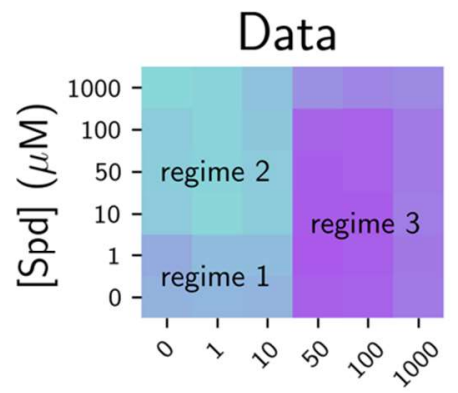
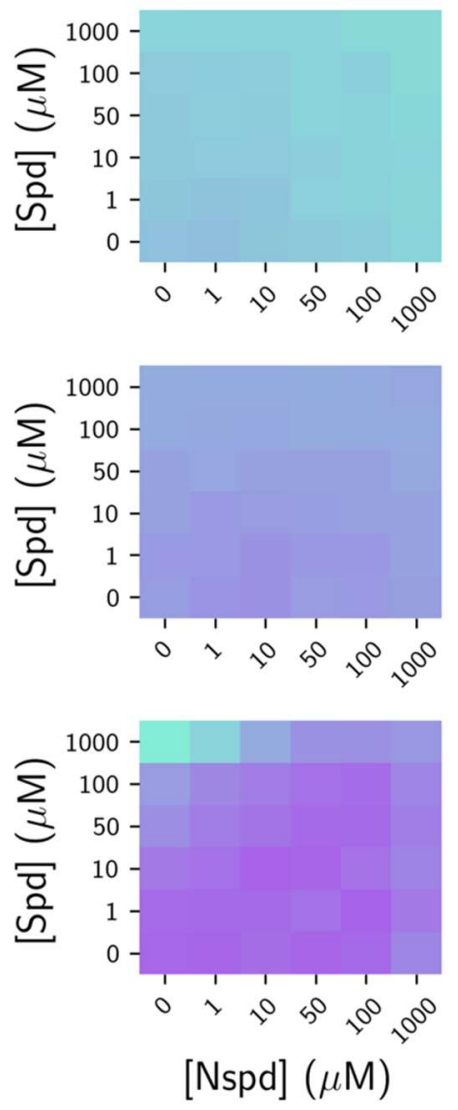
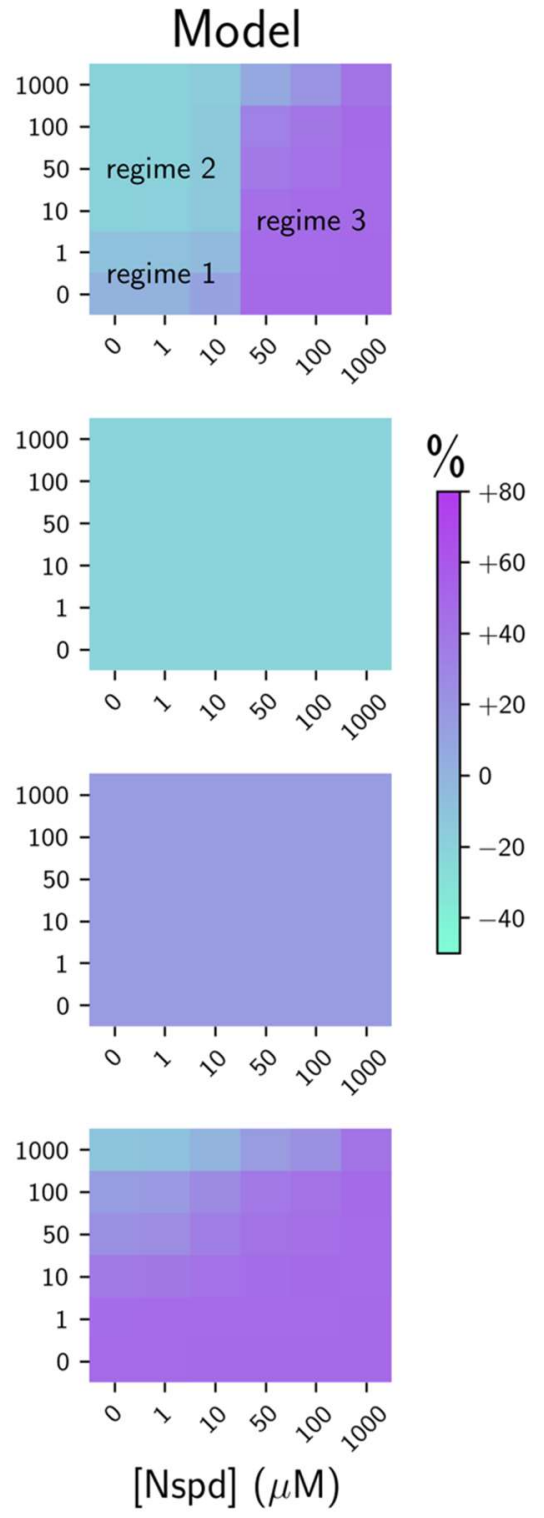

captures in vivo dynamics. (A) Scheme for the NspS-MbaA c-di-GMP polyamine signal transduction circuit. V. cholerae detects periplasmic norspermidine (Nspd) and spermidine (Spd) via the NspS-MbaA signaling system. Left panel: Detection of norspermidine by the periplasmic binding protein NspS drives its association with MbaA, promoting MbaA diguanylate cyclase activity and biofilm formation. Norspermidine is exported to the $V$. cholerae periplasm via an unknown mechanism and is reimported by the PotABCD1 system. Right panel: When norspermidine is absent, or when spermidine is detected, NspS dissociates from MbaA and MbaA functions as a c-di-GMP phosphodiesterase, biofilm formation is repressed, and biofilm dispersal is promoted. Norspermidine is produced by Vibrionaceae. Spermidine is a commonly produced polyamine but is not substantially produced by $V$. cholerae. $\mathrm{OM}=$ outer membrane; $I \mathrm{M}=$ inner membrane. In MbaA, the GGDEF and EAL domains have the sequences SGDEF and EVL, respectively. MbaA was previously shown to possess both diguanylate cyclase and phosphodiesterase activities (Bridges and Bassler, 2021). (B) Left panel: Schematic of the periplasmic polyamine sensing and import components in wildtype $V$. cholerae in the absence of exogenous polyamines. Middle panel: Experimentally obtained results for c-di-GMP reporter output in wildtype $V$. cholerae for the indicated polyamine concentrations, displayed as a heatmap. Throughout the manuscript, data in c-di-GMP output heatmaps are displayed as percent differences compared to the untreated wildtype strain, with teal representing low and purple representing high c-di-GMP reporter output, respectively. Right panel: Modeled c-di-GMP reporter output for wildtype $V$. cholerae based on a global fit to the experimental data for the wildtype, $\Delta n s p S, \Delta m b a A$, and $\Delta p o t D 1$ strains. (C) As in B for the $\Delta n s p S$ strain. (D) As in B for the $\Delta m b a A$ strain. (E) As in $B$ for the $\triangle$ potD1 strain. Experimental data are representative of 3 biological replicates. The experimental results shown in the middle panels in B-E are reproduced from (Bridges and Bassler, 2021), published under a Creative Commons Attribution license: https://creativecommons.org/licenses/by/4.0/.

To uncover the features of the NspS-MbaA signaling circuit that underpin the measured dose responses, we designed a free-energy model describing the relationship between

151 periplasmic polyamine concentrations and MbaA enzymatic activity. In the model, we propose

152 that norspermidine and spermidine influence MbaA activity exclusively through their effects on

153 the chemical equilibrium among NspS conformations. Specifically, we assume that NspS can

154 exist in one of two conformations: An "open" conformation, which is favored by spermidine binding

155 but which does not interact with MbaA, and a "closed" conformation, which is favored by

156 norspermidine binding and which drives the NspS-MbaA interaction. Notably, in the absence of

157 polyamine binding, an intrinsic free-energy offset between the NspS open and closed

158 conformations determines the equilibrium abundances of the two protein conformations. A given

159 MbaA receptor, in turn, can exist in any of three states: unbound to NspS and exhibiting

160 phosphodiesterase activity, unbound to NspS and exhibiting diguanylate cyclase activity, or 
161 bound to the closed conformation of $\mathrm{NspS}$ and exhibiting diguanylate cyclase activity. The

162 chemical equilibrium among the MbaA states determines the average activity across all MbaA

163 receptors and is calculated as a function of the free-energy offset between the phosphodiesterase

164 and diguanylate cyclase states, $f_{\text {MbaA }}$ (Methods). We incorporated the function describing MbaA

165 activity in a simple dynamical system considering polyamine fluxes into and out of the periplasm

166 and the kinetics of MbaA-driven c-di-GMP biosynthesis and degradation. To acquire values for

167 parameters, we fitted the model to the experimentally obtained c-di-GMP reporter data for the

168 wildtype, $\Delta n s p S, \Delta m b a A$, and $\Delta p o t D 1$ strains (Fig. 1B-E, middle panels). Our rationale for

169 choosing this particular model was that we could introduce experimentally titrated polyamine

170 concentrations as inputs to the model, compare the modeled steady-state c-di-GMP

171 concentrations to the experimentally obtained c-di-GMP reporter output data, and thus exploit the

172 model to obtain the parameters required to achieve the measured input-output dynamics. To

173 place additional constraints on the parameters, we experimentally measured the stoichiometry

174 (approximately 1:1) of functional NspS-3xFLAG and MbaA-FLAG proteins in vivo (Fig. S1A, B)

175 as well as the apparent dissociation constants of spermidine (48.4 $\pm 14.7 \mathrm{nM})$ and norspermidine

$176(67.1 \pm 12.2 \mathrm{nM})$ for purified NspS-6xHis using isothermal titration calorimetry (ITC) (Fig. S1C-

177 E).

178 Our model successfully captured the three regimes for the wildtype strain (Fig. 1B, right

179 panel), while achieving close fits to the data for the three mutants (Fig. 1C-E, right panels). The

180 model fits suggest that in wildtype $V$. cholerae, the first regime is explained by two factors: (1)

181 PotABCD1 imports norspermidine with high affinity $\left(K_{\mathrm{m}} \sim 1 \mathrm{nM}\right)$ and (2) only a small fraction of

182 NspS ( 0.7\%) exists in the closed state in the absence of norspermidine (Table 1). Supposition

183 (1) is supported by experimental results from the $\triangle$ potD1 strain, which show that in the absence

184 of polyamine import, elevated periplasmic norspermidine drives maximal MbaA-directed c-di-

185 GMP production (Fig. 1E, middle panel). Supposition (2) is supported by the $\Delta n s p S$ mutant 186 experimental data, which show that in the absence of NspS, the basal c-di-GMP level is lower 
187 than that of the wildtype (Fig. 1C, middle panel). Thus, in the first regime, the model predicts that

188 there is an inconsequentially low concentration of polyamines in the periplasm, but that a small

189 fraction of closed NspS nonetheless binds to MbaA and drives a modest level of c-di-GMP

190 production (Fig. 1B, right panel). In the second regime, our fitted kinetic parameters suggest that

191 PotABCD1-mediated import of spermidine is saturated, resulting in the accumulation of

192 periplasmic spermidine, which biases NspS to the open conformation (equivalent to an NspS-free

193 state, as in Fig. 1C). Therefore, this regime is explained by the intrinsic (NspS-free) fraction of

194 MbaA receptors that exist in the phosphodiesterase mode, which our fits suggest is around $75 \%$

195 (Table 1). In the third and final regime, our model suggests that the high concentration of

196 exogenously supplied polyamines saturates PotABCD1-mediated import, and therefore MbaA

197 activity is determined by a competition between periplasmic norspermidine and periplasmic

198 spermidine binding to NspS. Using our ITC measurements (Fig. S1) and the predicted fraction of

199 NspS that exists in the closed state (Table 1), we calculated the dissociation constant $K_{\text {nspd }}$ for

200 norspermidine for closed NspS to be $\sim 0.5 \mathrm{nM}$ and the dissociation constant $K_{\text {spd }}$ for spermidine

201 for open NspS to be $\sim 48.0 \mathrm{nM}$ (see Methods for details concerning these calculations). The

202 consequence of this $\sim 100$-fold difference in binding affinities is that once PotD1-mediated import

203 is saturated by norspermidine, the additional periplasmic norspermidine biases a significant

204 fraction of NspS toward the closed state, irrespective of the periplasmic concentration of

205 spermidine over the range studied (Fig. 1E, right panel). Thus, under this condition, MbaA acts

206 as a diguanylate cyclase. Together, these results suggest that the NspS-MbaA circuit functions

207 to maintain a basal cytoplasmic concentration of c-di-GMP via a mechanism in which a small

208 fraction of total NspS binds MbaA in the absence of exogenous norspermidine. This arrangement

209 may be necessary for the system to sensitively respond to both spermidine (and, in turn, degrade

210 c-di-GMP) and norspermidine (and, in turn, synthesize c-di-GMP). In addition, high affinity uptake

211 via PotABCD1 eliminates low-to-moderate concentrations of norspermidine and low 
212 concentrations of spermidine from the periplasm. The consequence is that under physiologically

213 realistic regimes, the concentrations of polyamines in the periplasm are sub-nanomolar.

\section{The mathematical model for NspS-MbaA signal transduction is predictive}

215 To test the fidelity of our model, we assessed its predictive capabilities by perturbing the

216 NspS-MbaA signal-transduction cascade. Specifically, we constructed a $V$. cholerae $\Delta m b a A$

217 strain harboring Pbad-mbaA, which allowed us to artificially alter $m b a A$ expression with arabinose.

218 We modulated MbaA production from non-detectable $(0 \%$ arabinose $)$ to modestly below that of

219 the wildtype ( $0.05 \%$ arabinose), to higher than wildtype levels $(0.2 \%$ arabinose $)$, as quantified by

220 Western blot (Fig. S2). We assessed how changing the level of MbaA influenced the steady-state

221 c-di-GMP concentration by measuring the c-di-GMP reporter output for each MbaA induction

222 condition and each polyamine concentration (Fig. 2, left panels). We used our model to predict

223 the c-di-GMP outputs under the same conditions (Fig. 2, right panels). As expected, in the

224 absence of arabinose, the experimental results and model predictions agreed (Fig. 2A), and

225 moreover, produced results akin to those for the $\triangle m b a A$ strain (Fig. 1D). Furthermore, our model

226 predictions achieved close fits to the experimental c-di-GMP reporter outputs for both MbaA

227 underexpression (Fig. 2B) and overexpression (Fig. 2C). Both under- and overexpression of

$228 \mathrm{MbaA}$ dampened the response to norspermidine relative to the wildtype, suggesting that the

229 NspS-MbaA circuit is maximally sensitive to changes in norspermidine levels near the

230 endogenous wildtype MbaA concentration and that norspermidine sensitivity decreases as MbaA

231 concentrations deviate from this level. Together, our results indicate that our mathematical

232 framework for the NspS-MbaA circuit accurately predicts the effects stemming from perturbations

233 to receptor levels, and that the natural system has evolved to be maximally sensitive to

234 norspermidine when there is import by PotABCD1. 


\section{Figure 2}

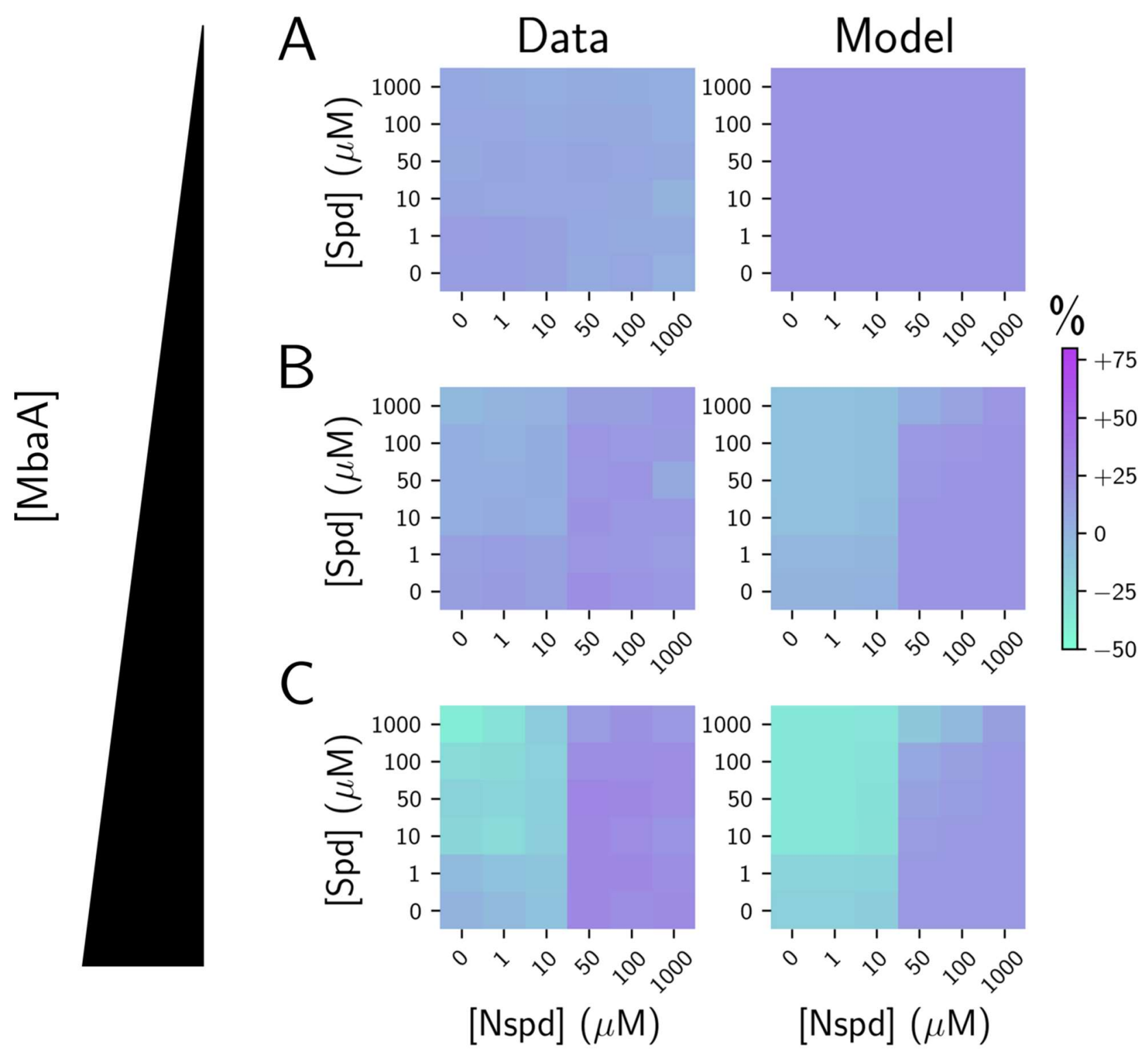

Fig. 2. The fitted mathematical model of NspS-MbaA input-output dynamics accurately predicts the outcomes of perturbations to MbaA protein levels. (A) Left panel: Experimentally obtained results for c-di-GMP reporter output in $\triangle m b a A \mathrm{~V}$. cholerae carrying Pbad-mbaA for the indicated polyamine concentrations at $0 \%$ arabinose, shown as a heatmap. Throughout the manuscript, data in c-di-GMP output heatmaps are displayed as percent differences compared to the untreated wildtype strain, with teal representing low and purple representing high c-di-GMP reporter output, respectively. Right panel: Modeled c-di-GMP reporter output for $\triangle m b a A V$. cholerae based on a global fit to the experimental data for the wildtype, $\Delta n s p S, \Delta m b a A$, and $\triangle$ potD1 strains. (B) Left panel: As in A, left panel, but with supplementation of $0.05 \%$ arabinose. Right panel: Modeled c-di-GMP reporter output for $V$. cholerae with $\sim 2.5$-fold lower MbaA levels than in wildtype $V$. cholerae. (C) Left panel: As in A, left panel, but with supplementation of $0.2 \%$ arabinose. Right panel: As in A, right panel, but for $\sim 1.5$-fold higher MbaA concentration than in wildtype $V$. cholerae. Experimental data are representative of 3 biological replicates. 


\section{The NspS-MbaA circuit is sensitive to sub-nanomolar periplasmic polyamine levels}

Our data show that PotABCD1-mediated polyamine import dramatically influences the ability of NspS-MbaA to detect and respond to polyamines in the periplasm (Fig. 1D). The consequence of rapid polyamine internalization is that polyamines (i.e., those supplied in

252 organisms in non-lab environments) are depleted from the periplasm. We reasoned that to 253 overcome ligand depletion by cytoplasmic import, the NspS-MbaA circuit must be exquisitely 254 sensitive to periplasmic polyamines. To test this supposition, we needed to quantify the function 255 of NspS-MbaA in a setup in which polyamine production and polyamine import were inactivated 256 to eliminate cell-driven changes to the fixed levels of polyamines we supplied exogenously. Our 257 strategy was to disable norspermidine production by deleting $n s p C$, the gene encoding the 258 carboxynorspermidine decarboxylase responsible for the final enzymatic step in norspermidine 259 production (Lee et al., 2009). To eliminate both polyamine production and import, we constructed 260 the $\triangle n s p C \triangle p o t D 1$ double mutant. Analysis of these two mutant phenotypes allowed us to assess 261 the intrinsic sensitivity of the NspS-MbaA circuit to periplasmic polyamines.

Regarding the $\triangle n s p C$ single mutant that is incapable of polyamine production: The model

263 predicts that the $V$. cholerae $\Delta n s p C$ mutant responses to external norspermidine and spermidine

264 resemble those of the wildtype (Fig. 3A, right panel). This outcome occurs because, as discussed 265 above, self-produced norspermidine exerts a negligible effect on basal MbaA activity in the 266 presence of polyamine import (Fig. 1A and B). Indeed, the middle panel of Fig. 3A shows that the $267 \Delta n s p C$ mutant exhibits the three c-di-GMP regimes displayed by the wildtype (see Fig. 1A), with 268 an $\mathrm{EC}_{50}$ for norspermidine of $\sim 10-50 \mu \mathrm{M}$. 
Figure 3
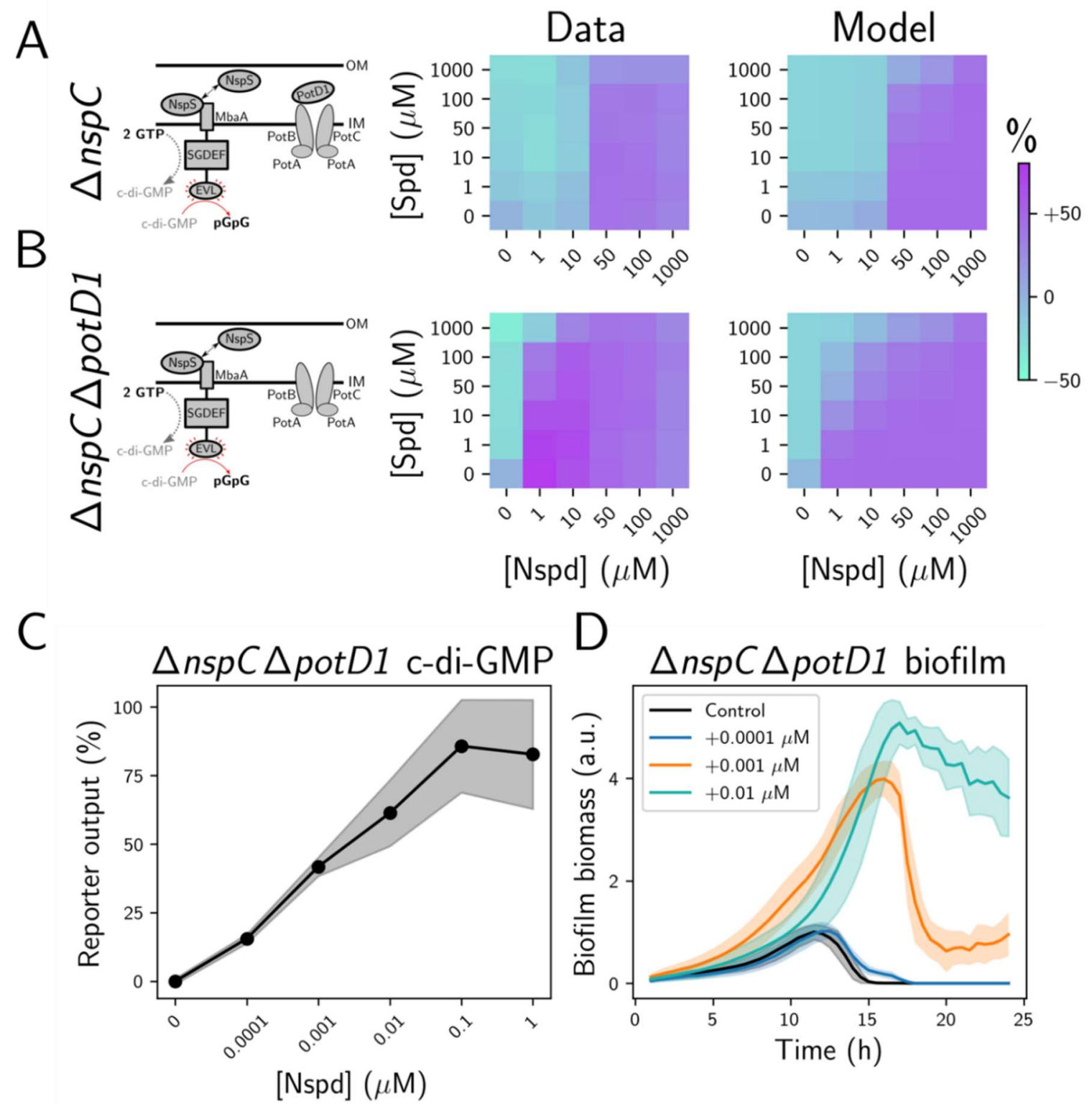

Fig. 3. MbaA-driven c-di-GMP production and biofilm formation are highly sensitive to norspermidine in the absence of PotD1-mediated import. (A) Left panel: Schematic of the periplasmic polyamine sensing and import components in $\triangle n s p C \mathrm{~V}$. cholerae in the absence of exogenous polyamines. Middle panel: Experimentally obtained results for the c-di-GMP reporter output, displayed as a heatmap, in $\triangle n s p C V$. cholerae for the indicated polyamine concentrations. $N=3$ biological replicates. Right panel: Modeled c-di-GMP reporter output for the $\Delta n s p C \mathrm{~V}$. cholerae strain. The parameters fitted to the data from Fig. 1 were used. (B) As in A for $\Delta n s p C$ $\triangle$ potD1 V. cholerae. Throughout the manuscript, data in c-di-GMP output heatmaps are displayed as percent differences compared to the untreated wildtype strain, with teal representing low and purple representing high c-di-GMP reporter output, respectively. C) Mean c-di-GMP output for $\triangle n s p C \triangle$ potD1 $\mathrm{V}$. cholerae at the specified norspermidine concentrations. $N=3$ biological replicates. (D) Biofilm biomass over time measured by bright field time-lapse microscopy for $\triangle n s p C \triangle$ potD1 $\mathrm{V}$. cholerae at the specified norspermidine concentrations. $N=3$ biological and $N=3$ technical replicates, \pm SD (shaded). a.u.: arbitrary unit. 
Regarding the $\triangle n s p C \triangle p o t D 1$ double mutant that is incapable of polyamine production and polyamine import: Our experimental results show that maximal c-di-GMP production was achieved at the lowest tested norspermidine concentration of $1 \mu \mathrm{M}$, and minimal c-di-GMP production occurred at $1 \mu \mathrm{M}$ spermidine treatment (Fig. 3B, middle panel). The consequence of sensitization to external polyamines is that the high c-di-GMP regime, in which norspermidine outcompetes spermidine, expands to include significantly lower norspermidine concentrations (Fig. 3B, middle panel). The model output agreed with the experimental results, showing that the $\triangle n s p C \triangle p o t D 1$ strain exhibits high sensitivity to exogenous spermidine and norspermidine relative to the $\triangle n s p C$ strain due to the elimination of periplasmic polyamine depletion by PotABCD1 (Fig.

292 3B, right panel). To define the intrinsic input-output relationship between periplasmic 293 norspermidine and c-di-GMP levels, we supplied norspermidine to the $\Delta n s p C \Delta p o t D 1$ strain at concentrations below $1 \mu \mathrm{M}$ and measured the reporter output. Fig. 3C shows that a response occurred at a norspermidine concentration as low as $0.1 \mathrm{nM}$, and the response was saturated by $100 \mathrm{nM}$. Consistent with these results, we calculated an $\mathrm{EC}_{50}$ for norspermidine of $\sim 1-5 \mathrm{nM}$ (Fig.

297 3C). Thus, in the absence of polyamine import and export, the NspS-MbaA circuit is sensitive to sub-nanomolar changes in periplasmic polyamine concentrations. Finally, the close agreement between the experimental results and the model for the $\Delta n s p C$ single and the $\Delta n s p C \Delta p o t D 1$ double mutants demonstrates that our model accurately captures the role of polyamine import in the response of the NspS-MbaA circuit to external polyamines. sensitive to changes in periplasmic norspermidine levels. To investigate whether such sensitivity 304 plays out via changes in downstream c-di-GMP-regulated behaviors, we assessed whether the $305 V$. cholerae $\triangle n s p C \triangle$ potD1 mutant also exhibited altered biofilm formation and dispersal in 306 response to sub-nanomolar concentrations of norspermidine. To do this, we administered $0.1 \mathrm{nM}$, $3071 \mathrm{nM}$, and $10 \mathrm{nM}$ norspermidine to the $\Delta n s p C \Delta$ potD1 strain and assayed biofilm formation by 
time-lapse microscopy. Indeed, a dose-dependent increase in biofilm biomass occurred in response to norspermidine (Fig. 3D). Together, these results show that sub-nanomolar levels of periplasmic norspermidine are detected by NspS-MbaA. Norspermidine detection leads to

311 increased MbaA diguanylate cyclase activity and cytoplasmic c-di-GMP accumulation, the result

312 of which is increased biofilm biomass accretion and reduced biofilm dispersal. Finally, the

313 consequence of PotABCD1-directed polyamine internalization in wildtype $V$. cholerae is that

314 cytoplasmic c-di-GMP levels are only altered in response to high concentrations of extracellular

315 norspermidine (>10 $\mu \mathrm{M}$; Fig. 1B, middle panel), despite the remarkable sensitivity of the NspS-

316 MbaA system to low concentrations of periplasmic polyamines.

\section{MbaA transmits information internally to elicit gene expression changes at polyamine concentrations well below those required to change the total concentration of cytoplasmic c-di-GMP}

NspS-MbaA detection of norspermidine and spermidine leads to induction and repression,

318 respectively, of vps biofilm matrix genes (Bridges and Bassler, 2021). However, the precise input-

319 output relationship between polyamine sensing, c-di-GMP levels, and downstream gene

320 expression is not known. To explore the relationship, we supplied exogenous norspermidine and

321 spermidine to wildtype $V$. cholerae carrying a vpsL-lux reporter and measured the

322 bioluminescence output. As shown above, in wildtype $V$. cholerae, exogenous norspermidine and

323 spermidine had no notable effects on measured c-di-GMP levels at low concentrations $(\leq 10 \mu M)$

324 (Fig. 1B, reproduced in Fig. 4A). By contrast, even at the lowest tested concentration ( $1 \mu \mathrm{M})$, both

325 polyamines drove significant changes in $v p s L$ expression (Fig. 4B). To determine if the changes

326 in vps expression tracked with changes in the biofilm lifecycle, we measured the biofilm biomass

327 over time. Consistent with the vpsL-lux data, biofilm biomass increased or decreased following

328 the addition of $1 \mu \mathrm{M}$ norspermidine or spermidine, respectively (Fig. 4C and D). Moreover, biofilm

329 dispersal was inhibited at $10 \mu \mathrm{M}$ exogenous norspermidine (Fig. 4D). As noted, changes in global

330 cytoplasmic c-di-GMP are only elicited when $50 \mu \mathrm{M}$ or higher polyamines are supplied (Fig. 4A 
331 and $\mathrm{E}$ ). Thus, the dichotomy is that vps gene expression and resulting changes in the $V$. cholerae

332 biofilm lifestyle are triggered at extracellular norspermidine and spermidine levels that have no

333 measurable effects on global cytoplasmic c-di-GMP levels. These results suggest that at low

334 polyamine concentrations, the NspS-MbaA circuit transmits c-di-GMP directly to transcription

335 factors via a local mechanism, the outcome of which is modulation of vps expression without

336 changes to global cytoplasmic c-di-GMP levels. By contrast, at high polyamine concentrations,

337 NspS-MbaA-mediated signaling alters both vps expression and the global cytoplasmic c-di-GMP

338 reservoir, which could potentially be accessed by all c-di-GMP effectors.

\section{Figure 4}

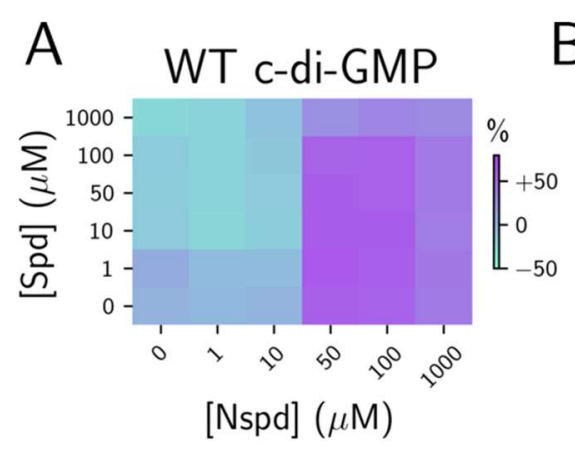

$\mathrm{D}$

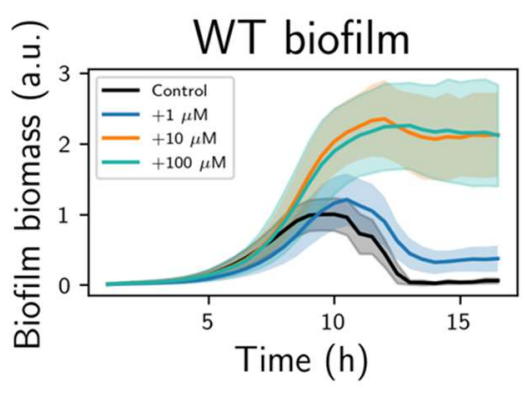

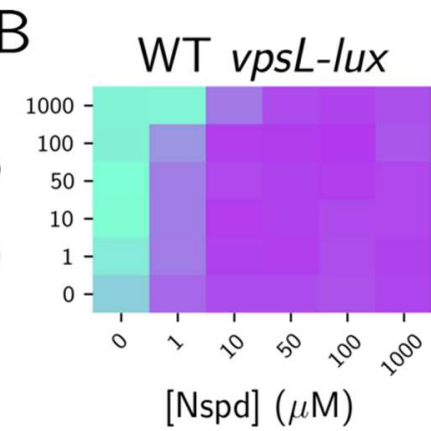

C

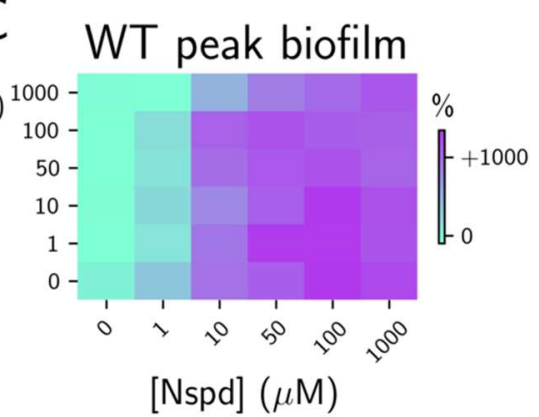

$\mathrm{E}$

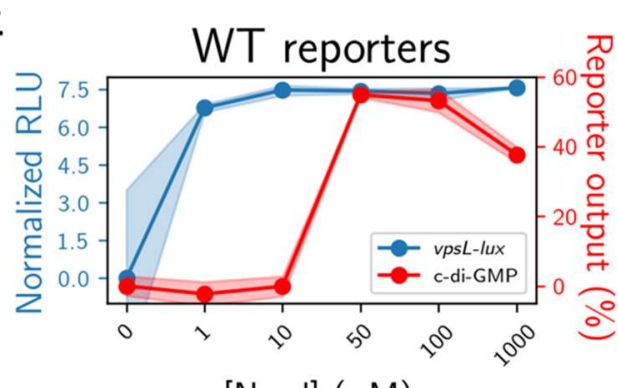

[Nspd] $(\mu \mathrm{M})$

Fig. 4. vps gene expression and biofilm formation are more sensitive to external polyamines than is the cytoplasmic c-di-GMP pool. (A) Experimentally obtained results for cdi-GMP reporter output in wildtype $V$. cholerae for the indicated polyamine concentrations, displayed as a heatmap and reproduced from Fig. 1A, middle panel. $N=3$ biological replicates. (B) As in (A) for vpsL-lux. Values are displayed as the $\log _{2}$ fold-changes relative to the untreated condition. (C) as in (B) for peak biofilm biomass measured by bright field time-lapse microscopy. $N=2$ biological and $N=2$ technical replicates. (D) Biofilm biomass over time measured by bright field time-lapse microscopy for wildtype $V$. cholerae at the specified norspermidine concentrations $N=3$ biological and $N=3$ technical replicates, \pm SD (shaded). a.u.: arbitrary unit. (E) Mean vpsLlux ( $\log _{2}$ fold-change relative to the untreated condition) and c-di-GMP outputs for wildtype $V$. cholerae at the specified norspermidine concentrations. These plots were generated from the data points in the bottom rows of panels $(A)$ and $(B)$. 


\section{NspS-MbaA signal transduction specifically controls expression of biofilm genes, not genes involved in other c-di-GMP-regulated processes}

352 transduction changes the cytoplasmic level of c-di-GMP, a diffusible second messenger known

353 to regulate a wide-variety of phenotypes, we wondered whether MbaA exclusively controls

354 transcription of biofilm genes or more generally affects the expression of genes involved in other

355 c-di-GMP-regulated processes, such as motility. To explore this question, we defined the NspS-

356 MbaA-controlled regulon by comparing the transcriptome of untreated wildtype $V$. cholerae to that

357 of wildtype $V$. cholerae treated with $100 \mu \mathrm{M}$ exogenous norspermidine (Fig. 5A, C) or $100 \mu \mathrm{M}$

358 spermidine (Fig. 5B, C). The data reveal that MbaA signaling is highly specific for control of biofilm

359 gene transcription; following norspermidine treatment, 20 of the 31 significantly upregulated

360 genes $\left(\log _{2}\right.$ fold-change $>1$ and $\mathrm{P}$-value $<0.05$ relative to untreated wildtype $V$. cholerae) encode

361 V. cholerae biofilm matrix genes, operons, or known biofilm regulators (e.g., vps-I, vps-II, rbm

362 cluster, and vpsT) (Fig. 5A, C, Supplementary Data 1). Consistent with this finding, spermidine

363 treatment caused downregulation of these same genes (20 of the 25 downregulated genes) (Fig.

$3645 B, C$, Supplementary Data 2). Expression of a few other genes was altered in response to the

365 polyamines, including $n s p C$, genes encoding some ribosome components, and some

366 transporters. Crucially, however, no genes involved in other known c-di-GMP-regulated

367 processes were significantly altered by polyamine treatment. We verified these findings by

368 performing the same analyses on the $\Delta m b a A$ mutant: Norspermidine and spermidine treatment

369 did not alter biofilm gene expression (Fig. 5C, Supplementary Data 3-5). Collectively, these data

370 demonstrate that the two polyamines regulate the $V$. cholerae biofilm lifecycle exclusively through

371 NspS-MbaA, and NspS-MbaA exerts an effect on only a small subset of c-di-GMP-responsive

372 genes. 
Figure 5

A

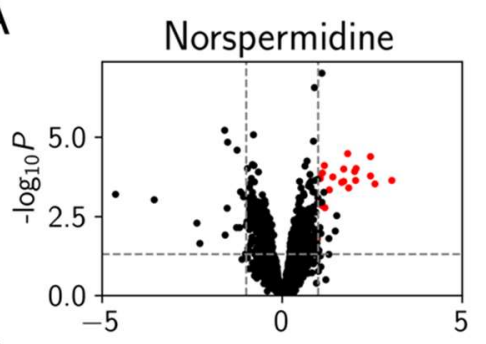

D

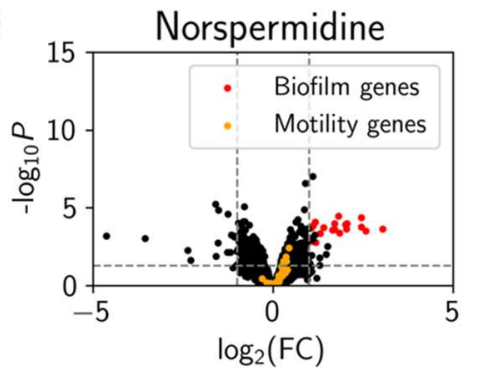

$\mathrm{F}$

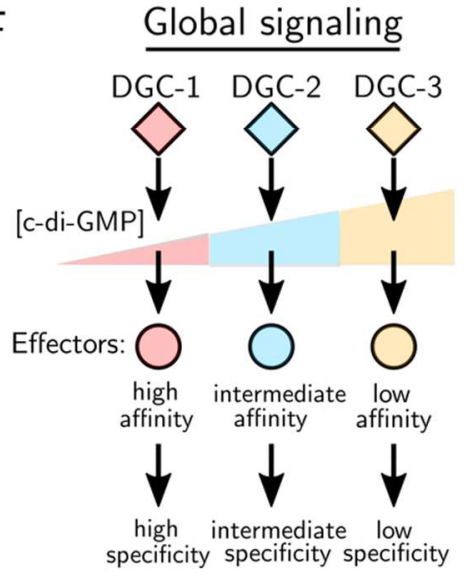

B

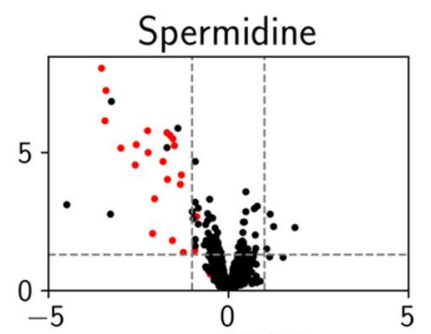

E
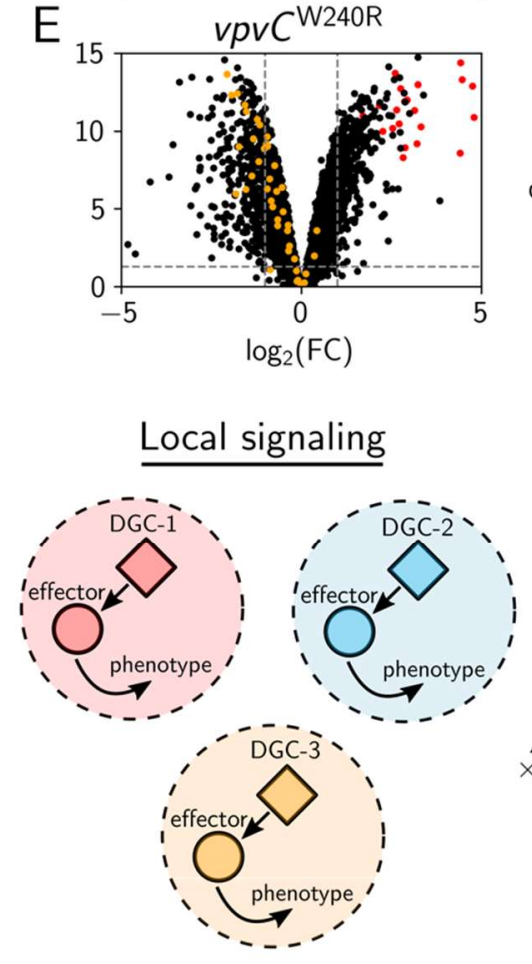

C

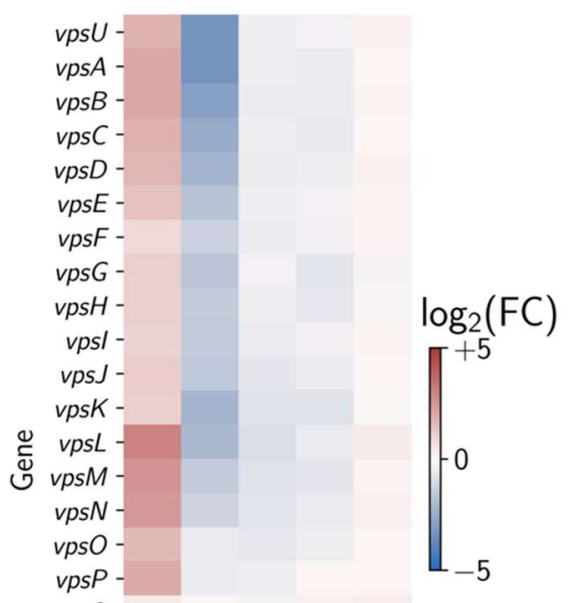

Fig. 5. Polyamine signaling through the NspS-MbaA circuit specifically controls biofilm gene expression. (A) Volcano plot showing fold changes (FC) in gene expression measured by RNA sequencing of the transcriptome of wildtype $V$. cholerae following administration of $100 \mu \mathrm{M}$ norspermidine relative to that of the untreated control. vps genes are highlighted in red, the horizontal dotted line represents a $p$-value of 0.05 and left and right vertical dashed lines represent $\log _{2}$ fold-changes of -1 and 1 , respectively. Samples were collected at $\mathrm{OD}_{600}=0.1$ and $N=3$ biological replicates. Complete datasets are available in the Supplemental Data. (B) As in A for $100 \mu \mathrm{M}$ spermidine. (C) Heatmap showing fold-changes in $V$. cholerae biofilm gene expression for the indicated treatments and strains relative to untreated wildtype $V$. cholerae (lanes 1-3) or the untreated $\triangle m b a A$ strain (lanes 4-5) $V$. cholerae. Red and blue represent increased and decreased gene expression levels, respectively. (D) Identical data as in panel A, re-scaled for ease of comparison to the $V$. cholerae $v p v C^{\mathrm{W} 240 \mathrm{R}}$ strain. $v p s$ genes are highlighted in red and motility genes are depicted in orange. (E) As in $D$ for the $V$. cholerae $v p v C^{\text {W240R }}$ strain. (F) Schematic comparing the proposed global (left panel) and local (right panel) models to achieve specificity in C-di-GMP signaling circuits. DGC: Diguanylate cyclase. See text for details. (Reviewed in Dahlstrom and O'Toole, 2017; Hengge, 2021). 
We wondered if the ability to drive changes in only a select subset of c-di-GMP-responsive outputs is unique to NspS-MbaA or if other $V$. cholerae c-di-GMP metabolizing enzymes regulate particular downstream genes with analogous selectivity. To address this question, we focused on specificity stemming from c-di-GMP synthesis by diguanylate cyclases. An especially attractive

393 feature of the NspS-MbaA system is that MbaA diguanylate cyclase activity can be ramped up via 394 exogenous supply of the norspermidine ligand. We are not aware of another individual V. cholerae 395 diguanylate cyclase that can be controlled with high specificity by administration of an identified 396 ligand. To overcome this issue, we exploited a V. cholerae strain that exhibits constitutive VpvC 397 diguanylate cyclase activity due to a point mutation (W240R) (Beyhan and Yildiz, 2007). The 398 mutation "locks" V. cholerae in biofilm-forming mode and dispersal does not occur. Thus, the $V$. 399 cholerae $v p v C^{W 240 R}$ mutant, at least phenotypically, mimics wildtype $V$. cholerae that has been 400 supplied with norspermidine. Unlike wildtype V. cholerae treated with norspermidine (Fig. 5D, 401 rescaled data from $5 \mathrm{~A}), \quad$. cholerae carrying $v p v C^{W 240 R}$ exhibited broad changes in gene 402 expression (725 genes were differentially expressed relative to wildtype) (Fig. 5E, and 403 Supplementary Data 6). The transcriptomic changes included higher activation of biofilm gene 404 expression than that following norspermidine treatment of wildtype (red points), and, additionally, 405 repression of genes involved in cell motility (orange points), and changes to hundreds of genes 406 required for other processes (black points). Thus, the c-di-GMP synthesized by VpvC ${ }^{\text {W240R }}$ causes 407 a dramatic and global reprogramming of $V$. cholerae gene expression, whereas the c-di-GMP 408 produced by MbaA exclusively regulates biofilm genes.

Two mechanisms have been proposed to account for specificity in the output of c-di-GMP-

410 responsive genes (Hengge, 2021; Massie et al., 2012). First is the "global signaling model" (Fig.

$4115 \mathrm{~F}$, left panel), in which the c-di-GMP produced by a given diguanylate cyclase freely diffuses

412 throughout the cytoplasm, and specificity in target gene expression is achieved entirely by 413 differences in affinities of downstream effectors for c-di-GMP (Hengge, 2021). For example, a 
414 weak diguanylate cyclase, represented by DGC-1 (Fig. 5F, left panel), produces a low level of c-

415 di-GMP. Only effectors with the highest affinities for c-di-GMP detect this change, and in turn,

416 alter genes or proteins in specific pathways. In our schematic, diguanylate cyclase DGC-2, which

417 makes more c-di-GMP than DGC-1, activates the DGC-1 effectors and additional lower-affinity

418 effectors. Consequently, DGC-2 elicits changes in the expression of a larger set of c-di-GMP-

419 regulated genes and the behaviors they specify than does DGC-1. Finally, a strong diguanylate

420 cyclase, represented by DGC-3, produces the highest level of c-di-GMP, activating all c-di-GMP-

421 responsive effectors, which in turn, drive large-scale changes in gene expression and behavior.

422 In this model, phosphodiesterases operate similarly except, when stimulated, they reduce the

423 global c-di-GMP pool. The second model, termed the "local signaling model", (Fig. 5F, right

424 panel), posits that diguanylate cyclases and phosphodiesterases convey information only to

425 particular downstream effectors, either through direct protein-protein interactions, or via other

426 means to produce local c-di-GMP pools (Hengge, 2021). In this model, specificity is achieved by

427 directly ferrying c-di-GMP from the diguanylate cyclase enzyme to the effector(s) or by local c-di-

428 GMP degradation in the case of phosphodiesterases.

\section{A global mechanism for c-di-GMP signaling delivers transcriptional specificity, but local c-di-GMP signaling via NspS-MbaA mediates increased polyamine sensitivity}

We considered whether local and/or global c-di-GMP signaling mechanisms could explain

430 the differences in transcriptional output specificity that occur due to c-di-GMP produced by NspS-

$431 \mathrm{MbaA}$ (highly specific) and that by $\mathrm{VpvC}^{\mathrm{W} 240 R}$ (non-specific). An obvious possibility in the context

432 of the global c-di-GMP signaling mechanism is that activation of MbaA by norspermidine

433 treatment only drives low-level production of cytoplasmic c-di-GMP, whereas VpvCW240R

434 generates higher c-di-GMP concentrations. Thus, c-di-GMP produced by MbaA only engages

435 high-affinity biofilm regulatory effectors, while c-di-GMP made by $\mathrm{VpvC}^{\mathrm{W} 240 \mathrm{R}}$ activates many more

436 effectors. To test this possibility, we used the c-di-GMP reporter to compare the levels of c-di-

437 GMP produced by the two diguanylate cyclases under the same conditions used for our 
438 transcriptomics measurements. The bottom panel of Figure 6 shows the results. Companion

439 transcriptomics results are displayed above those data in heatmaps, categorized by pathway.

440 Indeed, the $V$. cholerae $v p v C^{W 240 R}$ strain produced $\sim 2-3$-fold more c-di-GMP than wildtype $V$.

441 cholerae treated with norspermidine. Thus, it is possible that differences in cytoplasmic c-di-GMP

442 concentrations underpin the observed differences in gene expression outputs for the two

443 diguanylate cyclases.

444 In the context of our analyses, the global c-di-GMP signaling model makes two predictions:

445 (1) if MbaA-produced c-di-GMP were increased to match that of $\mathrm{VpvC}^{\mathrm{W} 240 \mathrm{R}}$, a broader set of gene

446 expression changes would occur, and specificity for biofilm regulation would be lost. (2)

447 Conversely, if $\mathrm{VpvC}^{\mathrm{W} 240 \mathrm{R}} \mathrm{c}$-di-GMP output were reduced to match that of MbaA following

448 norspermidine treatment, then $\mathrm{VpvC}^{\mathrm{W} 240 \mathrm{R}}$ would specifically regulate biofilm genes. To test

449 whether these predictions hold, we synthetically modulated the amount of c-di-GMP made by

$450 \mathrm{MbaA}$ and $\mathrm{Vpv} \mathrm{C}^{\mathrm{W} 240 \mathrm{R}}$, measured cytoplasmic c-di-GMP levels, and assessed the transcriptomes.

451 To address the first prediction, we increased c-di-GMP production by MbaA to match that of

$452 \mathrm{VpvC}^{\mathrm{W} 240 \mathrm{R}}$ by overexpressing $n s p S$ and $m b a A$ using the Ptac promoter (Fig. 6). Consistent with

453 the global model, $V$. cholerae overexpressing $n s p S$ and $m b a A$ no longer exhibited transcriptional

454 specificity. Rather, 624 genes were differentially regulated relative to wildtype. Notably, biofilm

455 genes showed strong activation and motility genes showed strong repression (Fig. 6, Fig. S3,

456 Supplemental Data 7). To address the second prediction, we controlled the expression of

$457 v p v C^{W 240 R}$ using the arabinose-controllable Pbad promoter (Fig. 6, Fig. S3, Supplemental Data

458 8). Overall, gene expression changed in step with the amount of $\mathrm{VpvC}{ }^{\mathrm{W} 240 \mathrm{R}}$ produced, as dictated

459 by the arabinose inducer concentration. At the arabinose concentration at which the c-di-GMP

460 output most closely matched that of wildtype $V$. cholerae treated with $100 \mu \mathrm{M}$ norspermidine, the

461 two strains demonstrated nearly identical transcriptomic profiles (Fig. 6, boxed). Unfortunately,

462 this inducible $\mathrm{VpvC}^{\mathrm{W} 240 \mathrm{R}}$ construct did not have sufficient dynamic range to enable us to achieve 
463 the high c-di-GMP levels required for repression of motility genes. Nonetheless, our results

464 suggest that the global c-di-GMP signaling model could explain the specificity of the NspS-MbaA

465 pathway for biofilm gene regulation and the lack of specificity in the $\mathrm{VpvC}^{\mathrm{W} 240 \mathrm{R}}$ strain.

466 The above findings suggest that any c-di-GMP diguanylate cyclase/phosphodiesterase

467 enzyme could deliver transcriptional specificity if its levels were appropriately modulated. To

468 determine if this is the case, we used our arabinose induction strategy to modulate the levels of

$469 \mathrm{CdgL}$, another V. cholerae diguanylate cyclase. We know that CdgL contributes to the basal c-di-

470 GMP pool because the $\Delta c d g L$ mutant possesses reduced cytoplasmic c-di-GMP and exhibits

471 lower biofilm gene expression than does wildtype $V$. cholerae. Indeed, the $\Delta c d g L$ mutant behavior

472 is akin to that of wildtype $V$. cholerae treated with $100 \mu \mathrm{M}$ spermidine (Fig. 6, Fig. S3,

473 Supplemental Data 9). We discovered cdgL induction conditions in which the cytoplasmic c-di-

474 GMP level equaled that of wildtype V. cholerae treated with $100 \mu \mathrm{M}$ norspermidine (Fig. 6, boxed).

475 Again, the transcriptional profile of the strain with synthetically modulated CdgL levels mirrored

476 that of wildtype following norspermidine treatment (Fig. 6, Fig. S3, Supplemental Data 9).

477 Together, these results show that c-di-GMP signaling specificity can be achieved exclusively

478 through changes to the global cytoplasmic pool. Thus, local signaling is not required for specificity

479 in transcription. 
bioRxiv preprint doi: https://doi.org/10.1101/2021.09.21.461265; this version posted September 21, 2021. The copyright holder for this preprint (which was not certified by peer review) is the author/funder, who has granted bioRxiv a license to display the preprint in perpetuity. It is made available under aCC-BY-NC 4.0 International license.

Figure 6

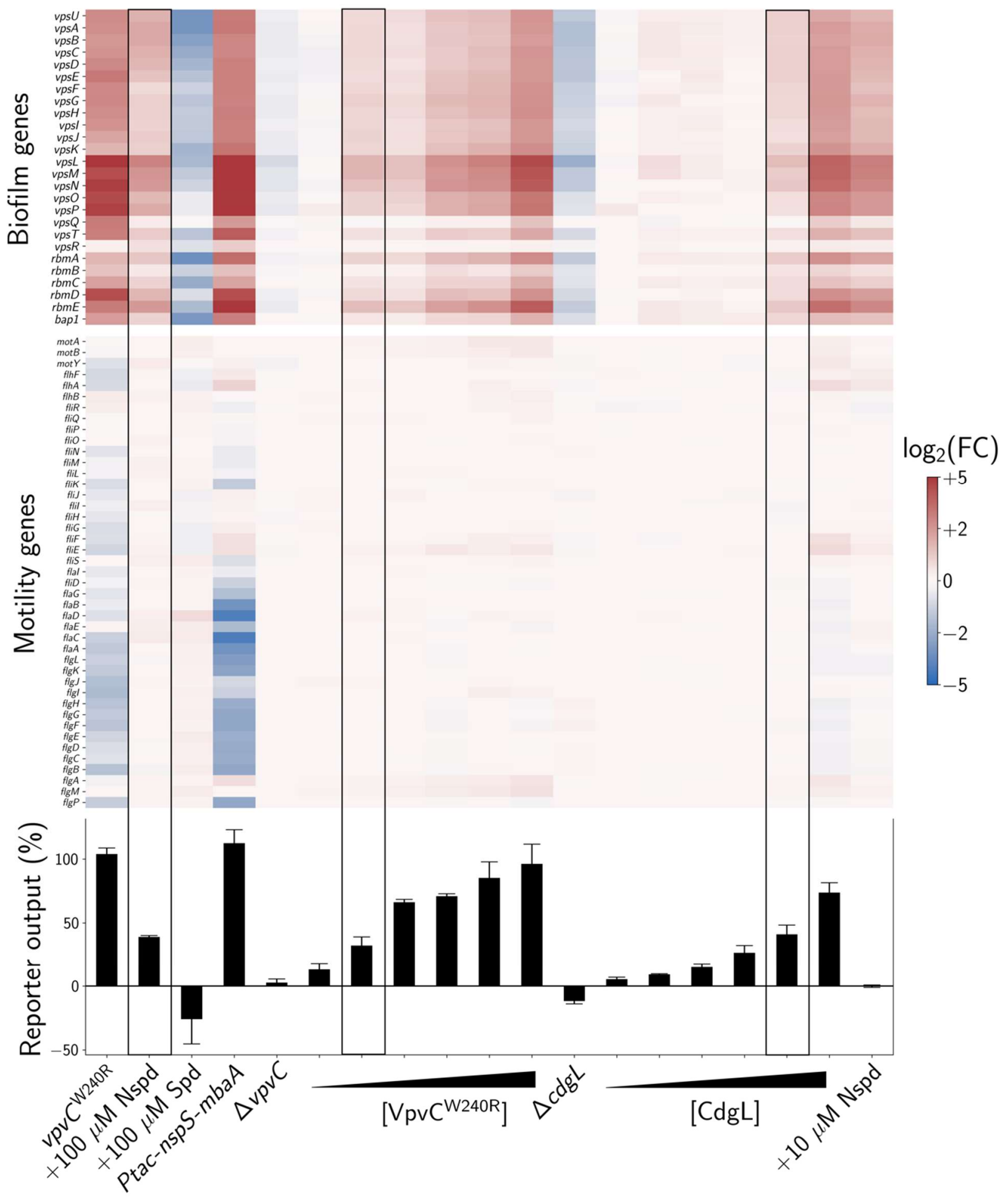


Fig. 6. Consequences of global and local c-di-GMP changes on $V$. cholerae gene expression patterns. Bottom panel: Mean global c-di-GMP reporter outputs for the indicated strains and conditions relative to untreated wildtype $V$. cholerae. $N=3$ biological replicates. Strains carrying cloned genes encoding enzymes that synthesize c-di-GMP were grown with $0 \%$, $0.0125 \%, 0.025 \%, 0.0375 \%, 0.05 \%$, or $0.1 \%$ arabinose to increasingly induce expression. To prevent excessive biofilm formation from interfering with reporter measurements, we deleted the $v p s L$ gene from the $v p v C^{W 240 R}$ and Ptac-nspS-mbaA strains. Data were normalized to the $\Delta v p s L$ parent strain carrying the c-di-GMP reporter. Top panel: Heatmap of $\log _{2}$ fold-changes for $V$. cholerae biofilm gene expression for the conditions and strains shown in the bottom panel. Genes are grouped by function (biofilm and motility), and red and blue represent increased and decreased expression levels, respectively. Boxed regions designate the gene expression outputs at approximately equal cytoplasmic c-di-GMP levels following norspermidine treatment, arabinose induction of $v p v C^{W 240 R}$, and arabinose induction of $c d g L$. Samples were collected at $O D_{600}=0.1$, complete datasets are available in the Supplemental Data, and volcano plots for each condition are shown in Fig. S3. For transcriptomics studies, the $v p v C^{W 240 R}$ and Ptac-nspS-mbaA strains carried intact vpsL. MbaA phosphodiesterase should be able to degrade c-di-GMP produced by a different enzyme, such as $\mathrm{VpvC}^{\mathrm{W} 240 \mathrm{R}}$. To test this possibility, we administered arabinose to $\mathrm{V}$. cholerae carrying Pbad-vpvC ${ }^{W 240 R}$ to achieve c-di-GMP reporter output equal to that of wildtype $V$. cholerae treated with $100 \mu \mathrm{M}$ norspermidine, as described above and shown in Fig. 6. We subsequently titrated in either norspermidine or spermidine and assessed whether, in response to the ligands, MbaA

502 altered the cytoplasmic concentration of c-di-GMP (Fig. S4). Indeed, norspermidine treatment

503 elevated the c-di-GMP level while spermidine treatment reduced the c-di-GMP level to roughly 504 that of untreated wildtype levels (Fig. S4). These results further demonstrate the global nature of 505 c-di-GMP signaling by showing that MbaA can contribute to and deplete the global cytoplasmic 506 c-di-GMP pool, the level of which is set by the combined activities of the suite of $V$. cholerae 507 diguanylate cyclases and phosphodiesterases. Collectively, our results suggest that in $V$. 508 cholerae, specificity in the biofilm gene expression output response to c-di-GMP signaling does 509 not require local c-di-GMP signaling and can be achieved via global changes in cytoplasmic c-di510 GMP levels (Fig. 6). 
The existence of global c-di-GMP signaling does not eliminate the possibility that local c-

512 di-GMP signaling could also take place. Indeed, our data indicate that MbaA transmits c-di-GMP

513 directly to select downstream biofilm effectors through a local mechanism, as evidenced by

514 activation of vpsL-Iux (Fig. 4) and other genes encoding components required for biofilm formation

515 (Fig. 6, Fig. S3, Supplemental Data 10) at norspermidine concentrations that are too low $(\leq 10$

$516 \mu \mathrm{M})$ to elicit increases to the global cytoplasmic c-di-GMP pool (Fig. 4). The proposed local

517 signaling mechanism employed by the NspS-MbaA circuit sensitizes $V$. cholerae to sub-

518 micromolar concentrations of polyamines. This feature of the NspS-MbaA system presumably

519 allows $V$. cholerae to modify its biofilm lifecycle in response to physiologically relevant polyamine

520 concentrations. Fig. 7 presents a schematic that reconciles the findings presented here and offers

521 a model for how ligand sensitivity and signaling specificity are achieved through both local and

522 global signaling.

\section{Figure 7}

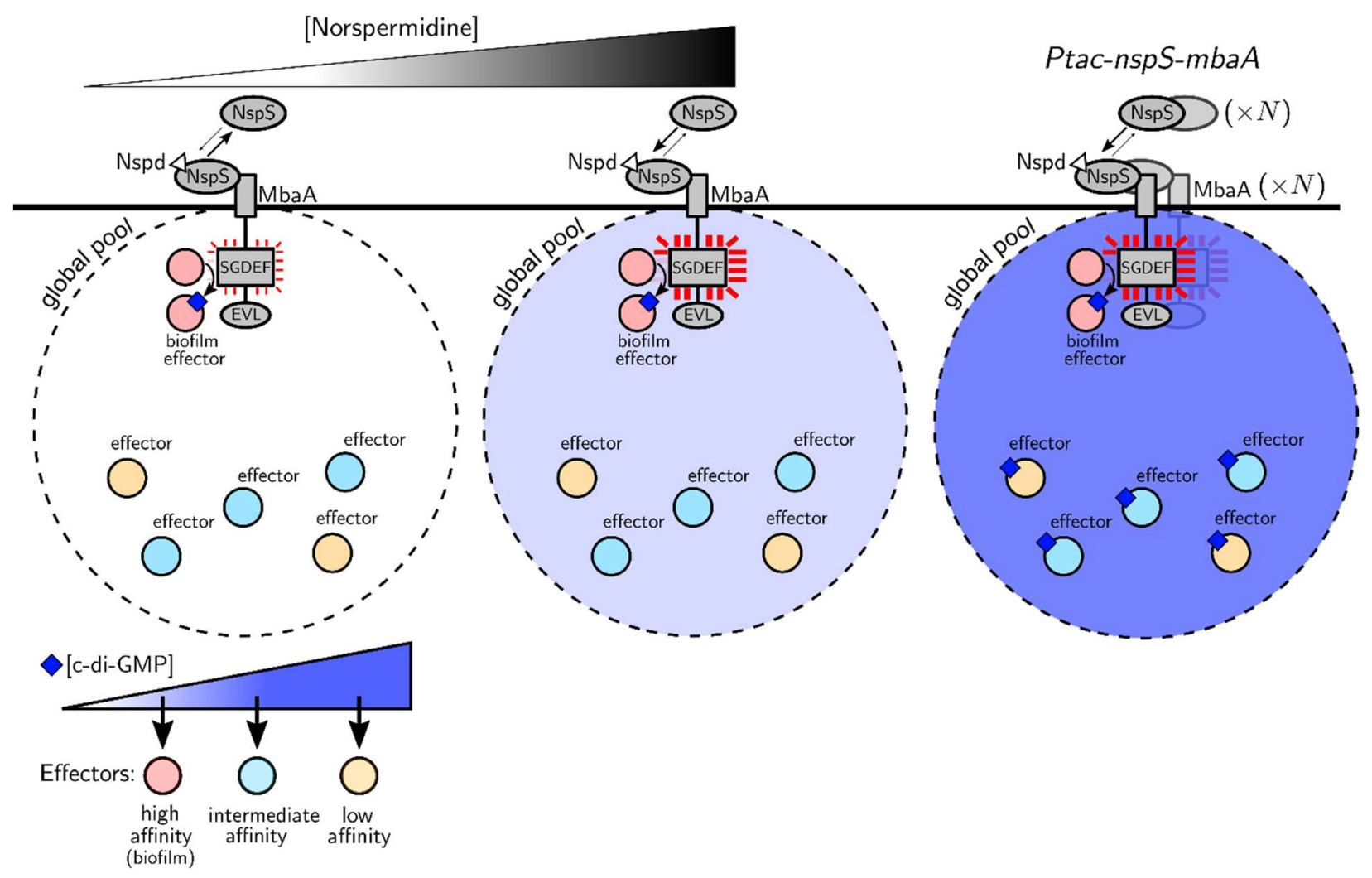



particular high affinity biofilm effectors via a local mechanism. This local mechanism sensitizes $V$. cholerae to norspermidine. At high norspermidine concentrations, when the NspS-MbaA diguanylate cyclase activity is maximal, the level of c-di-GMP that MbaA produces surpasses the amount that can be accommodated in the local pool. The extra c-di-GMP is contributed to the global pool and in principle becomes accessible to additional effectors. However, as shown in the results in Fig. 6, only the subset of transcription effectors with the highest affinity for c-di-GMP, i.e., those regulating biofilm gene expression, detect these low-level changes to the global c-diGMP pool. In the case of NspS-MbaA signaling, it appears that this subset of effectors is saturated by the local signaling mechanism. Thus, specificity in biofilm gene expression output is retained across all norspermidine ligand levels (Fig. 6). Under artificial conditions, such as overexpression of $n s p S$ and $m b a A$ by Ptac, the global c-di-GMP pool is driven higher, lower affinity effectors that control genes other than those involved in biofilms are engaged, and gene expression output 537 specificity is lost.

\section{Discussion}

In this study, we performed a quantitative analysis of signal transmission via the second-

541 output. The parameter values from our mathematical model, which are underpinned by

542 experimental data, suggest that high-affinity import depletes nearly all norspermidine from the

543 periplasm of wildtype V. cholerae. As a result, NspS is unliganded; however, the parameter values

544 also suggest that a small fraction of apo-NspS exists in the conformation that can bind MbaA,

545 resulting in slight $\mathrm{MbaA}$ diguanylate cyclase activity and consequently some production of

546 cytoplasmic c-di-GMP. If apo-NspS could not bind MbaA, MbaA would exhibit maximal

547 phosphodiesterase activity (akin to the experimental results from Fig. 1C), and V. cholerae would

548 not respond to fluxes of spermidine. On the other hand, if a large fraction of apo-NspS could bind

549 to $\mathrm{MbaA}, \mathrm{MbaA}$ would exhibit maximal diguanylate cyclase activity (akin to the experimental

550 results from Fig. 1E), and $V$. cholerae would not respond to fluxes in norspermidine. Thus, a key

551 takeaway from our modeling is that confining apo-NspS-MbaA complex formation to a low level

552 underlies the capacity of $V$. cholerae cells to respond to both norspermidine and spermidine. In

553 the future, the predictive capability of our model could be used, most powerfully in conjunction 
554 with genetic or synthetic biology approaches, to further characterize polyamine-mediated control

555 of $V$. cholerae biofilms.

$556 \quad$ Bacterial species frequently possess dozens of receptors harboring c-di-GMP biosynthetic

557 and catabolic activities, underscoring the widespread and conserved nature of these signaling

558 pathways (Conner et al., 2017). Because of the central importance of c-di-GMP-based regulation

559 of bacterial lifestyle decision making processes, c-di-GMP pathways have been proposed as

560 potential targets for the development of therapeutics that modify bacterial behavior (Trebino et

561 al., 2021). Success in such an endeavor could be accelerated by quantitative understanding of

562 the input-output relationships for specific c-di-GMP signaling circuits. A significant obstacle to

563 progress is that for the vast majority of c-di-GMP signaling systems, the ligands that stimulate the

564 receptors remain unknown (Jenal et al., 2017). Nevertheless, the framework presented here to

565 study the NspS-MbaA system can be adapted to other c-di-GMP signaling systems, even in the

566 absence of knowledge of the ligands, to motivate experimental approaches and perhaps hasten

567 targeting of c-di-GMP systems in biomedical applications.

568 In addition to the modeling effort, we used the NspS-MbaA pathway as a case study to

569 explore the roles of local and global c-di-GMP signaling pools. We find that c-di-GMP-driven

570 specificity in transcription of biofilm genes in $V$. cholerae does not require localized signaling, as

571 evidenced by our results following titrations of $\mathrm{VpvC}^{\mathrm{W} 240 R}$ and $\mathrm{CdgL}$ (Fig. 6). This result is

572 presumably a consequence of effectors with the highest affinity for c-di-GMP accessing the global

573 pool and, in turn activating transcription of biofilm genes (Fig. 4, 6, and 7). Nonetheless, our results

574 strongly imply that NspS-MbaA must also be capable of transmitting c-di-GMP directly to

575 particular effectors to alter downstream vps expression at low extracellular polyamine ligand

576 levels that are insufficient to affect the concentration of the global c-di-GMP pool (Fig. 7). Because

577 the output gene expression pattern is identical under conditions in which NspS-MbaA signals

578 exclusively locally (i.e., $10 \mu \mathrm{M}$ norspermidine) or locally and globally (i.e., when NspS-MbaA is

579 saturated at $100 \mu \mathrm{M}$ norspermidine), we presume that MbaA signals locally to the same set of 
580 effectors (i.e., those responsible for regulating biofilm genes) that detect changes to the global c-

581 di-GMP levels with high affinity. Thus, in the case of the NspS-MbaA system, while localized

582 signaling is not required for specificity, it does confer higher sensitivity to polyamines than could

583 be achieved if the relevant downstream effectors only responded to MbaA-driven changes to the

584 global c-di-GMP pool (Fig. 4 and 7). It is possible that in other c-di-GMP signaling systems, the

585 effectors responsible for conveying local signals differ from the high-affinity global effectors. In

586 such cases, output specificity, or lack thereof, could be governed by stimuli levels: At low stimulus

587 concentrations, specificity could be achieved through direct c-di-GMP transmission to a local

588 partner effector, analogous to the scenario we present for NspS-MbaA. However, at high stimulus

589 concentrations, the c-di-GMP produced could exceed the amount required to saturate the local

590 effectors, that c-di-GMP would leak into the global c-di-GMP pool, alter its levels, and drive broad

591 changes to gene expression. Thus, unlike for NspS-MbaA, output specificity would decline with

592 increasing stimulus concentration.

To prove the existence of the NspS-MbaA localized mechanism, it will be necessary to

594 define the downstream effectors that partner with NspS-MbaA. While not verified at present, we

595 suspect that the c-di-GMP responsive transcription factors $\mathrm{Vps} T$ and/or $\mathrm{VpsR}$ are the key

596 effectors and they are the focus of our ongoing work in this direction (Hsieh et al., 2018; Krasteva

597 et al., 2010). VpsT and VpsR are the master regulators of V. cholerae biofilm gene expression

598 and could interact with the MbaA catalytic domains (Teschler et al., 2015). In this regard, VpsT

599 could act doubly as a c-di-GMP effector as it is also known to regulate motility (Krasteva et al.,

600 2010). Direct interactions between c-di-GMP metabolizing enzymes and downstream effectors

601 have been demonstrated in other systems (Dahlstrom and O'Toole, 2017). It is also worth noting

602 that in the current work, we restricted our analyses to the transcriptional output in response to

603 NspS-MbaA-directed c-di-GMP signal transduction. It remains possible that NspS-MbaA also

604 controls c-di-GMP-dependent processes by post-transcriptional mechanisms, again acting either

605 locally or globally. 
It is increasingly appreciated that from bacteria to humans, second-messenger molecules

607 have the remarkable capacity to signal with high specificity despite their general use in an array

608 of processes in the same cell and in the face of their high diffusivity. Indeed, as one example,

609 calcium signaling in eukaryotes relies on many of the principles germane to c-di-GMP signaling

610 in bacteria: Calcium signal transduction lies at the core of cell physiology and function, there exist

611 multiple sources of the calcium messenger molecule, it is diffusible, and a large set of effectors

612 respond to changes in its levels (Bootman, 2012). A rich body of literature demonstrates that

613 calcium signaling fidelity is achieved via formation of local microdomains that promote directed

614 signal transmission (Berridge, 2006). Thus, in different guises, evolution has solved the same

615 issues associated with generically used, diffusible second-messenger signaling by devising

616 mechanisms for locally restricting signal transduction. 


\section{Methods}

\section{Model description}

618 To describe the relation between periplasmic polyamine concentrations and c-di-GMP

619 output from the NspS-MbaA circuit, we developed a two-state receptor model for MbaA. We

620 assume that MbaA exists in one of two states: the diguanylate cyclase state or the

621 phosphodiesterase state, which presumably correspond to distinct conformations of the MbaA

622 homodimer. The average phosphodiesterase activity of $\mathrm{MbaA},\left\langle A_{\mathrm{PDE}}\right\rangle$, is equivalent to the

623 probability of being in the phosphodiesterase state, which in the equilibrium statistical mechanical

624 description is determined exclusively by the free-energy offset between the phosphodiesterase

625 and diguanylate cyclase states, $f_{\mathrm{MbaA}}$ (with all energies in units of the thermal energy $k_{\mathrm{B}} T$ ):

$$
\left\langle A_{\mathrm{PDE}}\right\rangle=\frac{1}{1+\exp \left(f_{\mathrm{MbaA}}\right)} .
$$

As a consequence, the diguanylate cyclase activity of $\mathrm{MbaA},\left\langle A_{\mathrm{DGC}}\right\rangle$, is given by $\left\langle A_{\mathrm{DGC}}\right\rangle=$

$6271-\left\langle A_{\mathrm{PDE}}\right\rangle . f_{\mathrm{MbaA}}$ is a function of the intrinsic (NspS-free) free-energy offset between the

628 phosphodiesterase and diguanylate cyclase states, $\epsilon_{\mathrm{MbaA}}$, the concentration of free NspS in the

629 closed conformation, $N_{\text {free }}^{\text {closed }}$, and the binding constant of MbaA in the diguanylate cyclase state 630 for the closed conformation of NspS, $K_{\mathrm{MbaA}}$ :

$$
f_{\mathrm{MbaA}}=\epsilon_{\mathrm{MbaA}}+\log \left[1+\frac{N_{\text {free }}^{\text {closed }}}{K_{\mathrm{MbaA}}}\right] .
$$

$631 N_{\text {free }}^{\text {closed }}$, in turn, is a function of $\epsilon_{\mathrm{MbaA}}$ and $K_{\mathrm{MbaA}}$, as well as the intrinsic free-energy offset between

632 the open and closed states of apo-NspS $\left(\epsilon_{\mathrm{NspS}}\right)$, periplasmic norspermidine and spermidine

633 concentrations ( $n_{\text {peri }}$ and $s_{\text {peri }}$, respectively), the molar ratio of MbaA to NspS $(R)$, the binding

634 constants of each NspS conformation for norspermidine and spermidine ( $K_{\mathrm{nspd}}$ and $K_{\mathrm{spd}}$,

635 respectively), and the total concentration of NspS $(N)$ (see Supplementary Materials for the full 636 derivation): 


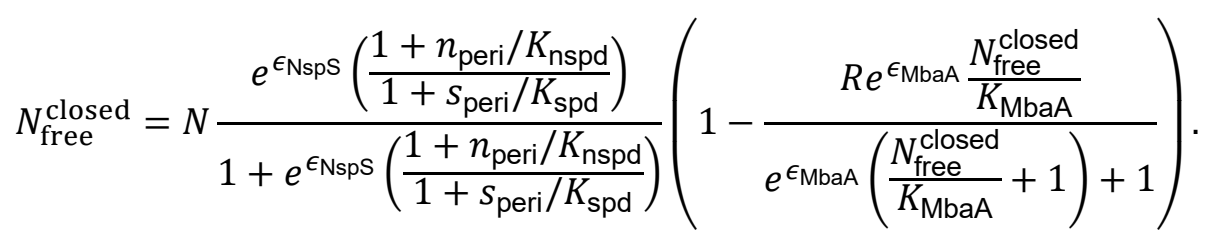

638 model the effect of a constant extracellular polyamine source on the steady-state proportions of

639 MbaA homodimers in the phosphodiesterase and diguanylate cyclase states and the effect of

640 MbaA activity on the cytoplasmic c-di-GMP concentration (for parameter definitions, see Table

641 1):

$$
\begin{gathered}
\frac{\mathrm{d} n_{\text {peri }}}{\mathrm{d} t}=\alpha+\beta_{n}\left(n_{\text {ext }}-n_{\text {peri }}\right)-\frac{\psi P n_{\text {peri }} / K_{\text {PotD1 }}^{n}}{1+\frac{s_{\text {peri }}}{K_{\text {PotD1 }}^{S}}+\frac{n_{\text {peri }}}{K_{\text {PotD1 }}^{n}}} \\
\frac{\mathrm{d} s_{\text {peri }}}{\mathrm{d} t}=\beta_{s}\left(s_{\text {ext }}-s_{\text {peri }}\right)-\frac{\varphi P s_{\text {peri }} / K_{\text {PotD1 }}^{S}}{1+\frac{S_{\text {peri }}}{K_{\mathrm{PotD} 1}^{S}}+\frac{n_{\text {peri }}}{K_{\text {PotD1 }}^{n}}} \\
\frac{\mathrm{d} c}{\mathrm{~d} t}=\gamma+\lambda\left\langle A_{\mathrm{DGC}}\right\rangle-\left(\mu+v\left\langle A_{\mathrm{PDE}}\right\rangle\right) c .
\end{gathered}
$$


Table 1: Model Parameters

\begin{tabular}{|c|c|c|}
\hline Symbol & Description & Value \\
\hline$N$ & Total concentration of NspS. & $16.7 \mu \mathrm{M}$ \\
\hline$R$ & Molar ratio of MbaA to NspS. & 1 \\
\hline$n_{\text {ext }}$ & Extracellular concentration of norspermidine. & Input \\
\hline$s_{\text {ext }}$ & Extracellular concentration of spermidine. & Input \\
\hline$K_{\text {spd }}$ & $\begin{array}{l}\text { Dissociation constant of spermidine from NspS in the open } \\
\text { conformation. }\end{array}$ & $48.0 \mathrm{nM}$ \\
\hline$K_{\text {nspd }}$ & $\begin{array}{l}\text { Dissociation constant of norspermidine from NspS in the closed } \\
\text { conformation. }\end{array}$ & $0.5 \mathrm{nM}$ \\
\hline$\epsilon_{\mathrm{NspS}}$ & $\begin{array}{l}\text { Free-energy offset between the open and closed conformations of } \\
\text { apo-NspS. }\end{array}$ & $-4.87 k_{\mathrm{B}} T$ \\
\hline$\epsilon_{\mathrm{MbaA}}$ & $\begin{array}{l}\text { Free-energy offset between the phosphodiesterase and diguanylate cyclase } \\
\text { states of MbaA in the absence of NspS. }\end{array}$ & $-1.214 k_{\mathrm{B}} T$ \\
\hline$K_{\mathrm{MbaA}}$ & $\begin{array}{l}\text { Dissociation constant of closed-conformation NspS from } \\
\text { diguanylate-cyclase-state MbaA. }\end{array}$ & $50 \mathrm{nM}$ \\
\hline$\psi$ & $\begin{array}{l}\text { Rate of norspermidine transport from the periplasm to the cytoplasm } \\
\text { by PotD1. }\end{array}$ & *10.6/t \\
\hline$\varphi$ & $\begin{array}{l}\text { Rate of spermidine transport from the periplasm to the cytoplasm } \\
\text { by PotD1. }\end{array}$ & $* 5.7 / t$ \\
\hline$P$ & Periplasmic concentration of PotD1. & $10 \mu \mathrm{M}$ \\
\hline$\alpha$ & Rate of norspermidine production and export to the periplasm. & $22.5 \mu \mathrm{M} / t$ \\
\hline$\beta_{n}$ & Rate of diffusion of extracellular norspermidine across the outer membrane. & $* 5 / t$ \\
\hline$\beta_{s}$ & Rate of diffusion of extracellular spermidine across the outer membrane. & $* 5 / t$ \\
\hline$K_{\text {PotD1 }}^{n}$ & Michaelis constant for PotD1-norspermidine. & $1 \mathrm{nM}$ \\
\hline$K_{\mathrm{PotD} 1}^{s}$ & Michaelis constant for PotD1-spermidine. & $434 \mathrm{nM}$ \\
\hline$\gamma$ & Background rate of c-di-GMP production. & $20 \mu \mathrm{M} / t$ \\
\hline$\nu$ & Background rate of c-di-GMP degradation. & $* 1 / t$ \\
\hline$\mu$ & Rate of c-di-GMP degradation by MbaA. & $* 0.78 / t$ \\
\hline$\lambda$ & Rate of c-di-GMP synthesis by MbaA. & $7.8 \mu \mathrm{M} / t$ \\
\hline
\end{tabular}

${ }^{*}$ In this table, parameter values with units of inverse time $1 / t$ are denoted value $/ t$, where $t$ is an arbitrary unit of time.

\section{Model fitting procedure}

To fit the mathematical model to our experimentally obtained c-di-GMP reporter assay

643 data, we set $n_{\text {ext }}$ and $s_{\text {ext }}$ equal to the experimentally supplied concentrations of norspermidine

644 and spermidine, respectively, initialized the state variables, and simulated (3-5) to a steady state

645 over a range of parameter values. To optimize the parameter values, we used nonlinear least

646 squares, which seeks a minimizing vector of parameter values, $\boldsymbol{x}^{*}$, for a nonlinear objective

647 function, $F$, of the form 


$$
\min _{\boldsymbol{x}} F(\boldsymbol{x}) \equiv \min _{\boldsymbol{x}} \frac{1}{2}\|r(\boldsymbol{x})\|_{2}^{2}=\min _{\boldsymbol{x}} \frac{1}{2} \sum_{i=1}^{m} r_{i}(\boldsymbol{x})^{2}
$$

648 where $r_{i}(\boldsymbol{x})$ are the residuals representing the offset between each measured data point $\left(y_{i}\right)$ and

649 the modeled steady-state c-di-GMP output $\left(c^{*}\right)$ when the supplied norspermidine and spermidine 650 concentrations are $n_{i}$ and $s_{i}$, respectively:

$$
r_{i}(\boldsymbol{x})=y_{i}-q c^{*}\left(\boldsymbol{x}, n_{i}, s_{i}\right)
$$

In (8), the model c-di-GMP output is scaled by a constant $q$, because the c-di-GMP

652 reporter responds linearly with cytoplasmic c-di-GMP concentration (Zhou et al., 2016). To

653 perform this optimization task, we implemented the Levenberg-Marquardt algorithm through the

654 Imfit package in Python 3 (Van Rossum and Drake Jr, 1995) (see Supplementary Materials for

655 further details). The fitted parameter values are shown in Table 1.

\section{Calculating the $K_{\mathrm{d}}$ values for the different NspS conformations from ITC measurements}

We calculated the binding affinity of norspermidine for the closed conformation of NspS

$657\left(K_{\mathrm{nspd}}\right)$ based on the equilibrium fraction of NspS bound to norspermidine. In general, in the 658 absence of spermidine, this fraction is given by

$$
p_{\text {bound }}=\frac{e^{\epsilon_{\mathrm{Nsps}}} \frac{n_{\text {peri }}}{K_{\mathrm{nspd}}}}{1+e^{\epsilon_{\mathrm{Nsps}}\left(\frac{n_{\text {peri }}}{K_{\mathrm{nspd}}}+1\right)}}
$$

659 where $\epsilon_{\mathrm{NspS}}$ denotes the intrinsic free-energy offset between the open and closed conformations

660 of NspS. Thus, the relation between the measured apparent binding affinity, $K_{d}^{n}$, and the closed-

661 conformational binding affinity, is given by

$$
K_{\mathrm{d}}^{n}=\frac{K_{\mathrm{nspd}}\left(1+\exp \left(\epsilon_{\mathrm{Nsps}}\right)\right)}{\exp \left(\epsilon_{\mathrm{NspS}}\right)}
$$


663 and the binding affinity of spermidine for open NspS, $K_{\mathrm{spd}}$, is given by

$$
K_{\mathrm{d}}^{S}=K_{\mathrm{spd}}\left(1+\exp \left(\epsilon_{\mathrm{Nsps}}\right)\right)
$$

\section{Bacterial strains, reagents, reporters, imaging assays, and western blots}

The wildtype $V$. cholerae parent used in this work was $V$. cholerae $01 \mathrm{El}$ Tor biotype C6706str2. All strains used in this work are reported in Table S1. When necessary, antimicrobials were supplied at the following concentrations: polymyxin $\mathrm{B}, 50 \mu \mathrm{g} / \mathrm{mL}$; kanamycin, $50 \mu \mathrm{g} / \mathrm{mL}$;

667 spectinomycin, $200 \mu \mathrm{g} / \mathrm{mL}$; chloramphenicol, $10 \mu \mathrm{g} / \mathrm{mL}$; and gentamicin, $15 \mu \mathrm{g} / \mathrm{mL}$. Strains used

668 for cloning were propagated on lysogeny broth (LB) plates supplemented with $1.5 \%$ agar or in 669 liquid LB with shaking at $30^{\circ} \mathrm{C}$. Strains used in reporter quantitation, biofilm assays, and RNA 670 isolation were grown in M9 minimal medium with $0.5 \%$ dextrose and $0.5 \%$ casamino acids.

671 Norspermidine (Millipore Sigma, I1006-100G-A), spermidine (Millipore Sigma, S2626-1G), and

672 arabinose (Millipore Sigma, W325501) were added at the concentrations designated in the figures

673 or figure legends at the start of each assay. c-di-GMP, vpsL-lux, and biofilm biomass over time

674 were measured as previously described (Bridges and Bassler, 2019, 2021; Bridges et al., 2020).

675 The heatmap of peak biofilm biomass in Fig. 4 employed brightfield images that were obtained

676 using a Biotek Cytation 7 imaging plate reader and a 20x objective lens. All plots were generated

677 using Python 3 (Van Rossum and Drake Jr, 1995). Western blotting of MbaA-3xFLAG and NspS-

678 3xFLAG was performed as described previously (Bridges and Bassler, 2021).

\section{DNA manipulation and strain construction}

Modifications to the $V$. cholerae genome were generated by replacing genomic DNA with

680 linear DNA introduced by natural transformation as described previously (Bridges et al., 2020).

681 PCR and Sanger sequencing (Genewiz) were used to verify results. See Table S2 for primers

682 and g-blocks (IDT) used in this study. Due to overlap between the $n s p S$ and $m b a A$ coding regions, 
gene synthesis was used to simultaneously produce nspS-3xFLAG at the endogenous locus and

684 preserve the downstream coding sequence of $m b a A$. To achieve this arrangement, the nspS-

$685 m b a A$ overlapping region was duplicated. The nspS codon usage was altered in the upstream

686 duplication while preserving the amino acid sequence, and the 5'-most mbaA start codon was

687 disabled by mutation. DNA encoding $3 x F L A G$ was introduced immediately upstream of

688 the $n s p S$ stop codon. DNA specifying a flexible linker (5x glycine, serine repeats) was inserted

689 between the DNA encoding the 3' terminus of NspS and the 5' start of the DNA encoding the

$6903 x$ FLAG tag. We found that such a linker was required for function. Arabinose titratable Pbad

691 expression constructs were introduced at the neutral locus, vc1807. Reporters expressed on

692 plasmids were introduced into V. cholerae strains via conjugation with E. coli S17 $\lambda$ pir.

\section{NspS protein purification and isothermal titration calorimetry}

DNA encoding NspS-6xHis, excluding its secretion signal (residues 1-34), was cloned into

694 the pET-15b vector using Gibson assembly (NEB). Production of NspS-6xHis protein was initiated

695 in E. coli BL21 (DE3) by the addition of 1 mM IPTG, followed by growth with shaking for $20 \mathrm{~h}$ at

$69618^{\circ} \mathrm{C}$. The cells were pelleted at $16,000 \times \mathrm{g}$ for $20 \mathrm{~min}$ and resuspended in lysis buffer (50 mM

697 Tris- $\mathrm{HCl} \mathrm{pH}$ 8.0, $150 \mathrm{mM} \mathrm{NaCl}, 10 \mathrm{mM}$ imidazole, $1 \mathrm{mM}$ DTT, $0.2 \mathrm{mg} / \mathrm{mL}$ lysozyme, $25 \mathrm{u} / \mathrm{mL}$

698 benzonase nuclease, and 1x EDTA-free protease inhibitor cocktail (Roche)). The cells were lysed

699 using sonication and subjected to centrifugation at 32,000 x $g$ for $30 \mathrm{~min}$. NspS-6xHis protein was

700 purified from the clarified supernatant using Ni-NTA Superflow resin (Qiagen) equilibrated in lysis

701 buffer. The column was washed three times with 10x column volumes of wash buffer (50 mM Tris-

$702 \mathrm{HCl} \mathrm{pH} \mathrm{8.0,300} \mathrm{mM} \mathrm{NaCl,} 20$ mM Imidazole, $1 \mathrm{mM}$ DTT, and 1x EDTA-free protease inhibitor

703 cocktail) and the protein was eluted with the same buffer except containing $300 \mathrm{mM}$ imidazole.

704 The eluate was immediately concentrated and subjected to a Superdex-200 size exclusion

705 column (GE Healthcare) in gel filtration buffer (reduced salt PBS - $1.8 \mathrm{mM} \mathrm{KH}_{2} \mathrm{PO}_{4}, 10 \mathrm{mM}$ 
$\mathrm{Na}_{2} \mathrm{HPO}_{4}, 2.7 \mathrm{mM} \mathrm{KCl}$, and $75 \mathrm{mM} \mathrm{NaCl}$ ). Peaks containing the highest purity protein were collected, and the protein was stored on ice and subjected to ITC as soon as possible.

The binding affinities of polyamines to NspS-6xHis were measured using a MicroCal PEAQ-ITC (Malvern) instrument at $25^{\circ} \mathrm{C}$. Norspermidine and spermidine were each dissolved in

710 the above gel filtration buffer. To measure $K_{d}$ values, the indicated concentrations of each

711 polyamine were titrated into a solution containing $7 \mu \mathrm{M}$ apo-NspS-6xHis with continuous stirring

712 at $750 \mathrm{rpm}$. The instrument was controlled with PEAQ-ITC Control software (MicroCal) and results

713 were fitted and evaluated by the PEAQ-ITC Analysis software (MicroCal).

\section{RNA isolation and sequencing}

Overnight cultures of the indicated $V$. cholerae strains, grown in biological triplicate, were

715 diluted to $\mathrm{OD}_{600} \sim 0.001$ in $5 \mathrm{~mL}$ of fresh $\mathrm{M} 9$ medium. These sub-cultures were grown at $30^{\circ} \mathrm{C}$

716 with shaking in the presence of the designated polyamine and/or arabinose to $O D_{600}=0.1$. Cells

717 were harvested by centrifugation for $10 \mathrm{~min}$ at $3200 \mathrm{x} \mathrm{g}$ and resuspended in RNAprotect (Qiagen).

718 RNA was isolated using the RNeasy mini kit (Qiagen), remaining DNA was digested using the

719 TURBO DNA-free kit (Invitrogen), and the concentration and purity of RNA were measured using

720 a NanoDrop instrument (Thermo). Samples were flash frozen in liquid nitrogen and stored at -

$72180^{\circ} \mathrm{C}$ until they were shipped on dry ice to the Microbial Genome Sequencing Center (MIGS;

722 https://www.migscenter.com/rna-sequencing). Upon sample submission, the 12 million paired-

723 end reads option and the intermediate analysis package were selected for each sample. As per

724 the MIGS project report, quality control and adapter trimming were performed with bcl2fastq

725 (Illumina), while read mapping was performed with HISAT2 (Kim et al., 2019). Read quantitation

726 was performed using the Subread's featureCounts (Liao et al., 2014) functionality, and

727 subsequently, counts were loaded into R ( $R$ Core Team) and normalized using edgeR's

728 (Robinson et al., 2010) Trimmed Mean of M values (TMM) algorithm. Values were converted to 729 counts per million (cpm) and differential expression analyses were performed using edgeR's 
730 Quasi-Linear F-Test (qlfTest) functionality against treatment groups, as indicated. Heatmaps and

731 volcano plots were produced in Python 3 (Van Rossum and Drake Jr, 1995).

\section{Data and code availability}

732 All data generated in this study and codes used for data analysis and plotting are included in the

733 manuscript and supporting files.

\section{Acknowledgements}

We thank the members of the Bassler and Wingreen groups for insightful comments and ideas. Isothermal Titration Calorimetry was performed in the Princeton Biophysics Core Facility with Venu Vandavasi. This work was supported by the Howard Hughes Medical Institute (B.L.B.); the National Science Foundation through the Center for the Physics of Biological Function (PHY1734030), as well as NSF grants MCB-1713731 (B.L.B.) and MCB-1853602 (B.L.B. and N.S.W.); NIH grants 1R21Al144223 (B.L.B. and N.S.W.), 2R37GM065859 (B.L.B.), GM082938 (N.S.W.), and 1K99Al158939 (A.A.B); and the Max Planck Society-Alexander von Humboldt Foundation (B.L.B.). During this work, A.A.B. was a Howard Hughes Medical Institute Fellow of the Damon Runyon Cancer Research Foundation (DRG-2302-17). 


\section{$\underline{\text { References }}$}

Berridge, M.J. (2006). Calcium microdomains: organization and function. Cell Calcium 40, 405412.

Beyhan, S., and Yildiz, F.H. (2007). Smooth to rugose phase variation in Vibrio cholerae can be mediated by a single nucleotide change that targets c-di-GMP signalling pathway. Mol Microbiol 63, 995-1007.

Bootman, M.D. (2012). Calcium signaling. Cold Spring Harb Perspect Biol 4, a011171.

Bridges, A.A., and Bassler, B.L. (2019). The intragenus and interspecies quorum-sensing autoinducers exert distinct control over Vibrio cholerae biofilm formation and dispersal. PLoS Biol. 17, e3000429.

Bridges, A.A., and Bassler, B.L. (2021). Inverse regulation of Vibrio cholerae biofilm dispersal by polyamine signals. eLife 10, e65487.

Bridges, A.A., Fei, C., and Bassler, B.L. (2020). Identification of signaling pathways, matrixdigestion enzymes, and motility components controlling Vibrio cholerae biofilm dispersal. PNAS 117, 32639-32647.

Cockerell, S.R., Rutkovsky, A.C., Zayner, J.P., Cooper, R.E., Porter, L.R., Pendergraft, S.S., Parker, Z.M., McGinnis, M.W., and Karatan, E. (2014). Vibrio cholerae NspS, a homologue of ABC-type periplasmic solute binding proteins, facilitates transduction of polyamine signals independent of their transport. Microbiology 160, 832-843.

Conner, J.G., Zamorano-Sánchez, D., Park, J.H., Sondermann, H., and Yildiz, F.H. (2017). The ins and outs of cyclic di-GMP signaling in Vibrio cholerae. Curr Opin Microbiol 36, 20-29.

Dahlstrom, K.M., and O'Toole, G.A. (2017). A symphony of cyclases: specificity in diguanylate cyclase signaling. Annu Rev Microbiol 71, 179-195.

Dow, J.M., Fouhy, Y., Lucey, J.F., and Ryan, R.P. (2006). The HD-GYP domain, cyclic di-GMP signaling, and bacterial virulence to plants. Mol Plant Microbe Interact 19, 1378-1384.

Flemming, H.-C., and Wingender, J. (2010). The biofilm matrix. Nat Rev Microbiol 8, 623-633.

Flemming, H.-C., Wingender, J., Szewzyk, U., Steinberg, P., Rice, S.A., and Kjelleberg, S. (2016). Biofilms: an emergent form of bacterial life. Nat Rev Microbiol 14, 563-575.

Guilhen, C., Forestier, C., and Balestrino, D. (2017). Biofilm dispersal: multiple elaborate strategies for dissemination of bacteria with unique properties. Mol Microbiol 105, 188-210.

Hengge, R. (2021). High-specificity local and global c-di-GMP signaling. Trends Microbiol 0.

Hsieh, M.-L., Hinton, D.M., and Waters, C.M. (2018). VpsR and cyclic di-GMP together drive transcription initiation to activate biofilm formation in Vibrio cholerae. Nucleic Acids Res 46, 88768887. 
Jenal, U., Reinders, A., and Lori, C. (2017). Cyclic di-GMP: second messenger extraordinaire. Nat Rev Microbiol 15, 271-284.

Karatan, E., Duncan, T.R., and Watnick, P.I. (2005). NspS, a predicted polyamine sensor, mediates activation of Vibrio cholerae biofilm formation by norspermidine. J Bacteriol 187, 74347443.

Kim, D., Paggi, J.M., Park, C., Bennett, C., and Salzberg, S.L. (2019). Graph-based genome alignment and genotyping with HISAT2 and HISAT-genotype. Nat Biotechnol 37, 907-915.

Krasteva, P.V., Fong, J.C.N., Shikuma, N.J., Beyhan, S., Navarro, M.V.A.S., Yildiz, F.H., and Sondermann, H. (2010). Vibrio cholerae VpsT regulates matrix production and motility by directly sensing cyclic di-GMP. Science 327, 866-868.

Lee, J., Sperandio, V., Frantz, D.E., Longgood, J., Camilli, A., Phillips, M.A., and Michael, A.J. (2009). An alternative polyamine biosynthetic pathway is widespread in bacteria and essential for biofilm formation in Vibrio cholerae. J Biol Chem 284, 9899-9907.

Liao, Y., Smyth, G.K., and Shi, W. (2014). featureCounts: an efficient general purpose program for assigning sequence reads to genomic features. Bioinformatics 30, 923-930.

Mah, T.-F., Pitts, B., Pellock, B., Walker, G.C., Stewart, P.S., and O'Toole, G.A. (2003). A genetic basis for Pseudomonas aeruginosa biofilm antibiotic resistance. Nature 426, 306-310.

Massie, J.P., Reynolds, E.L., Koestler, B.J., Cong, J.-P., Agostoni, M., and Waters, C.M. (2012). Quantification of high-specificity cyclic diguanylate signaling. PNAS 109, 12746-12751.

McGinnis, M.W., Parker, Z.M., Walter, N.E., Rutkovsky, A.C., Cartaya-Marin, C., and Karatan, E. (2009). Spermidine regulates Vibrio cholerae biofilm formation via transport and signaling pathways. FEMS Microbiol Lett 299, 166-174.

R Core Team R: A language and environment for statistical computing.

Robinson, M.D., McCarthy, D.J., and Smyth, G.K. (2010). edgeR: a Bioconductor package for differential expression analysis of digital gene expression data. Bioinformatics 26, 139-140.

Römling, U., Galperin, M.Y., and Gomelsky, M. (2013). Cyclic di-GMP: the first 25 years of a universal bacterial second messenger. Microbiol Mol Biol Rev 77, 1-52.

Shaw, T., Winston, M., Rupp, C.J., Klapper, I., and Stoodley, P. (2004). Commonality of elastic relaxation times in biofilms. Phys Rev Lett 93, 098102.

Simm, R., Morr, M., Kader, A., Nimtz, M., and Römling, U. (2004). GGDEF and EAL domains inversely regulate cyclic di-GMP levels and transition from sessility to motility. Mol Microbiol 53, 1123-1134.

Sobe, R.C., Bond, W.G., Wotanis, C.K., Zayner, J.P., Burriss, M.A., Fernandez, N., Bruger, E.L., Waters, C.M., Neufeld, H.S., and Karatan, E. (2017). Spermine inhibits Vibrio cholerae biofilm formation through the NspS-MbaA polyamine signaling system. Journal of Biological Chemistry 292, 17025-17036. 
Teschler, J.K., Zamorano-Sánchez, D., Utada, A.S., Warner, C.J.A., Wong, G.C.L., Linington, R.G., and Yildiz, F.H. (2015). Living in the matrix: assembly and control of Vibrio cholerae biofilms. Nat Rev Microbiol 13, 255-268.

Trebino, M.A., Shingare, R.D., MacMillan, J.B., and Yildiz, F.H. (2021). Strategies and approaches for discovery of small molecule disruptors of biofilm physiology. Molecules 26, 4582.

Van Rossum, G., and Drake Jr, F.L. (1995). Python tutorial.

Young, E.C., Baumgartner, J.T., Karatan, E., and Kuhn, M.L. (2021). A mutagenic screen reveals NspS residues important for regulation of Vibrio cholerae biofilm formation. Microbiology.

Zamorano-Sánchez, D., Xian, W., Lee, C.K., Salinas, M., Thongsomboon, W., Cegelski, L., Wong, G.C.L., and Yildiz, F.H. (2019). Functional specialization in Vibrio cholerae diguanylate cyclases: distinct modes of motility suppression and c-di-GMP production. mBio 10, e00670-19.

Zhou, H., Zheng, C., Su, J., Chen, B., Fu, Y., Xie, Y., Tang, Q., Chou, S.-H., and He, J. (2016). Characterization of a natural triple-tandem c-di-GMP riboswitch and application of the riboswitchbased dual-fluorescence reporter. Sci Rep 6, 20871. 
Figure S1
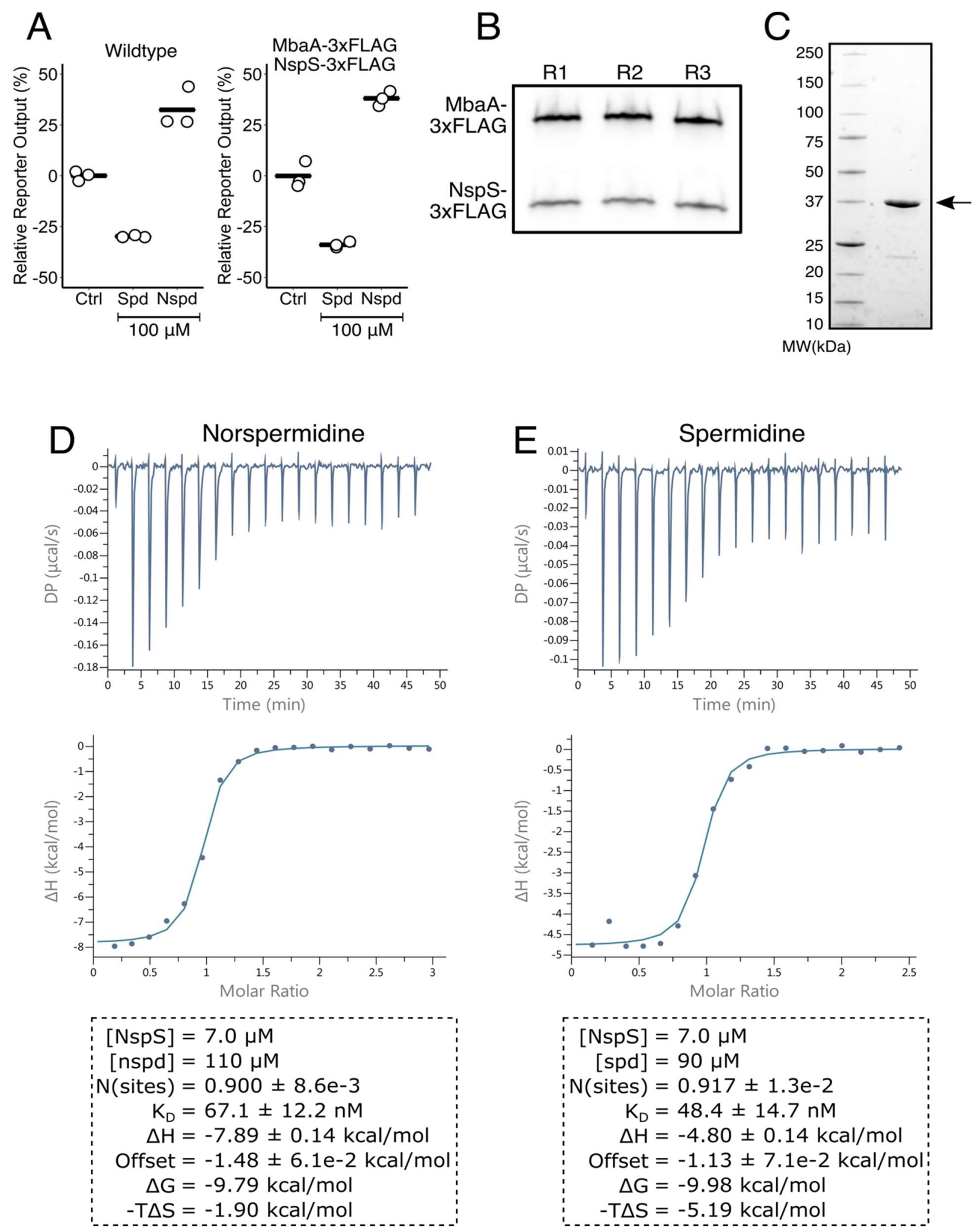
Fig. S1. In vivo stoichiometry of NspS and MbaA proteins and ITC measurements of binding constants. (A) Relative c-di-GMP reporter output for wildtype $V$. cholerae (left panel) and $V$. cholerae harboring $m b a A-3 x-F L A G$ and $n s p S-3 x F L A G$ (right panel) expressed from the native locus on the chromosome. Treatments: No addition (designated Ctrl), $100 \mu \mathrm{M}$ spermidine, and $100 \mu \mathrm{M}$ norspermidine. (B) Western blot of MbaA-3xFLAG and NspS-3xFLAG. R1, R2, and R3 designate three biological replicates. (C) SDS-PAGE gel showing purity of the NspS-6xHis protein used for ITC measurements. Molecular weight markers are designated on the left. The arrow on the right shows the position of NspS-6x-His. (D) ITC data, plot, and calculated values for norspermidine binding to purified NspS-6xHis. (E) As in $D$ for spermidine. 
bioRxiv preprint doi: https://doi.org/10.1101/2021.09.21.461265; this version posted September 21, 2021. The copyright holder for this preprint (which was not certified by peer review) is the author/funder, who has granted bioRxiv a license to display the preprint in perpetuity. It is made available under aCC-BY-NC 4.0 International license.

Figure $\mathbf{S 2}$

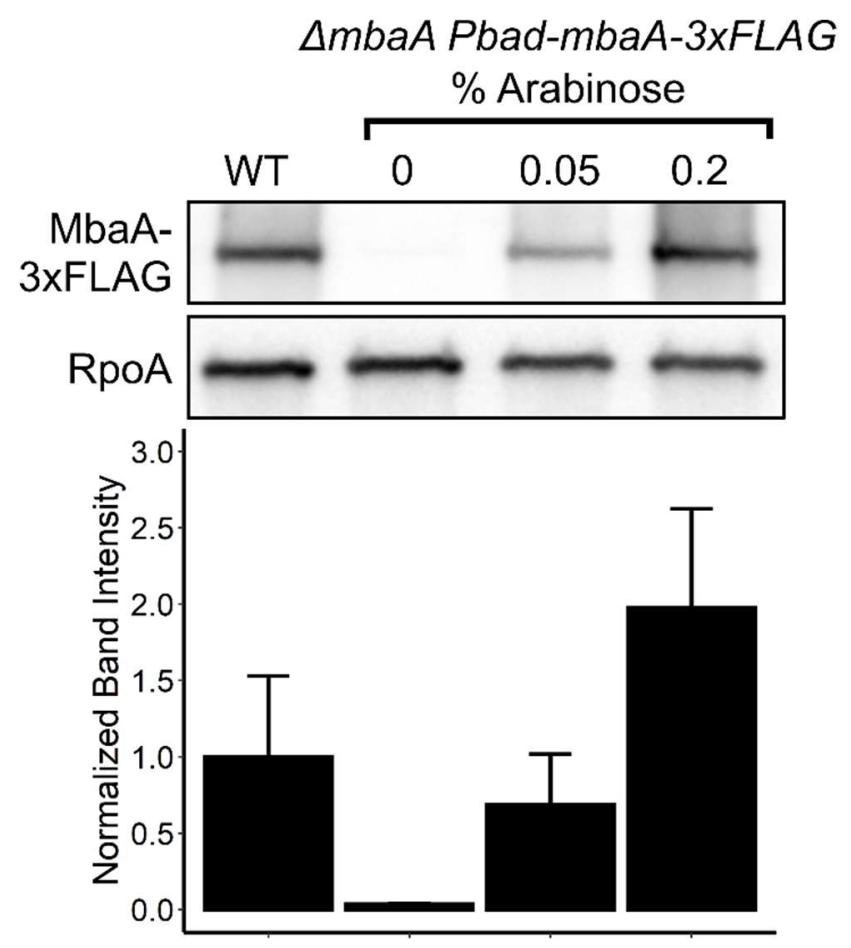

Fig. S2. MbaA-3xFLAG levels can be controlled by arabinose and quantified. Representative western blot (top) and companion quantitation (bottom) of MbaA-3xFLAG levels for $V$. cholerae carrying $m b a A-3 x F L A G$ at the endogenous mbaA locus (referred to as WT, leftmost lane and companion bars), and for the $V$. cholerae $\triangle m b a A$ Pbad-mbaA-3xFLAG strain following induction by the indicated arabinose concentrations (right three lanes and companion bars). RpoA was used as the loading control in the western blot. 
Figure S3
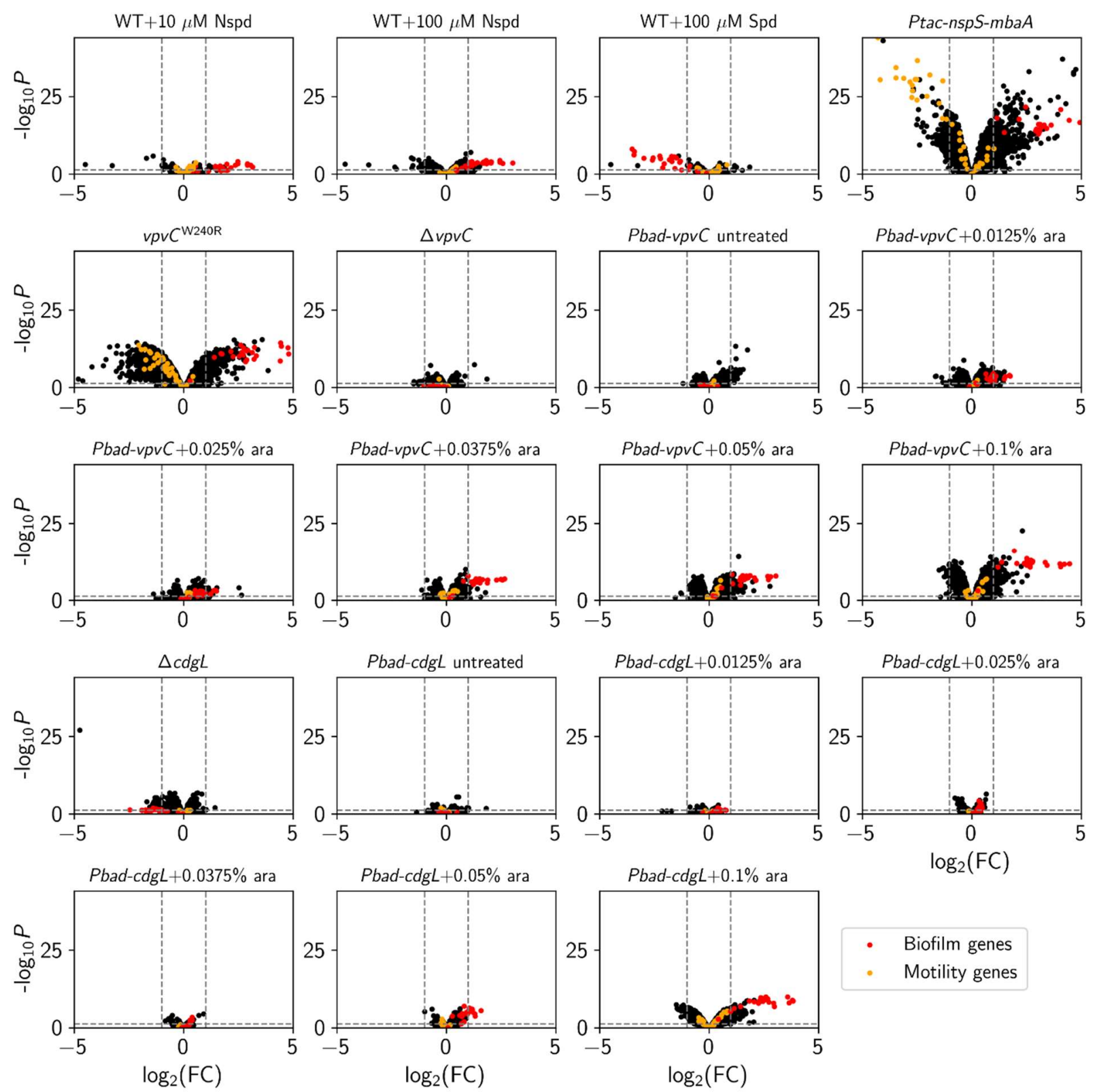

Biofilm genes

Motility genes

Fig. S3. Biofilm gene expression specificity can be achieved by altering the global cytoplasmic c-di-GMP level. Volcano plots showing fold-changes (FC) in gene expression relative to the corresponding controls, measured by RNA sequencing. Fold-changes were quantified relative to untreated wildtype $V$. cholerae for all conditions except those involving arabinose, which were compared to the wildtype strain treated with the equivalent arabinose concentration. Plots represent the full transcriptional profiles for the strains and conditions shown in Fig. 6. Biofilm genes are highlighted in red and motility genes are depicted in orange. The horizontal dotted line represents a $p$-value of 0.05 and left and right vertical dashed lines represent $\log _{2}$ fold-changes of -1 and 1 , respectively. Samples were collected at $\mathrm{OD}_{600}=0.1$ and $N=3$ biological replicates. Complete datasets are available in the Supplemental Data. 


\section{Figure S4}

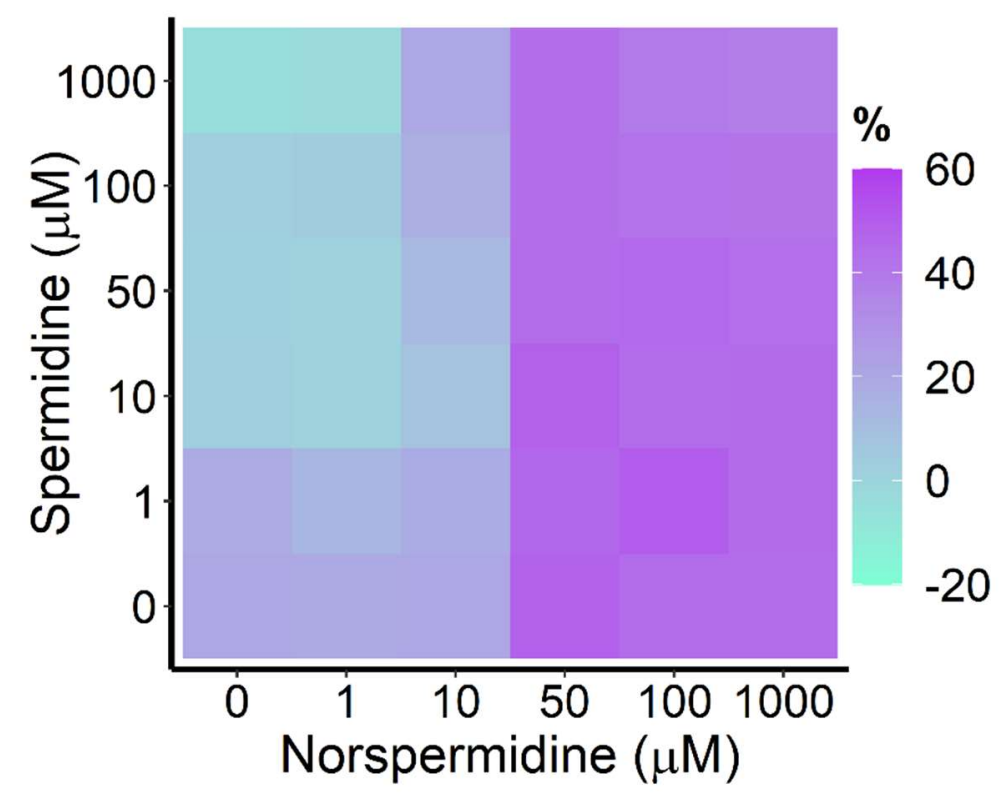

Fig. S4. The NspS-MbaA system can modify the c-di-GMP pool established by VpvC ${ }^{\text {W240R. }}$ Experimentally obtained results for c-di-GMP reporter output in $V$. cholerae carrying Pbad$v p v C^{W 240 R}$ treated with $0.0125 \%$ arabinose, for the indicated polyamine concentrations, displayed as a heatmap. Throughout the manuscript, data in c-di-GMP output heatmaps are displayed as percent differences compared to the untreated wildtype strain, with teal representing low and purple representing high c-di-GMP reporter output, respectively. 
Table S1. Strains used in this study.

\begin{tabular}{|c|c|c|c|c|}
\hline Strain Number & Genotype & Plasmid & $\begin{array}{l}\text { Antibiotic } \\
\text { Resistance }\end{array}$ & Origin \\
\hline BB_Vc_0090 & Wildtype O1 El Tor biotype C6706str2 & - & Sm & $\begin{array}{l}\text { Laboratory } \\
\text { wildtype }\end{array}$ \\
\hline AB_Vc_761 & $\begin{array}{l}\Delta v c 1807:: \mathrm{Cm}^{\mathrm{R}} \text { (Referred to as wildtype in this } \\
\text { work) }\end{array}$ & - & $\mathrm{Sm}, \mathrm{Cm}$ & $\begin{array}{l}\text { Bridges et al. } \\
2020\end{array}$ \\
\hline AB_Vc_956 & Wildtype & pFY4357::Gm & $\mathrm{Sm}, \mathrm{Gm}$ & $\begin{array}{l}\text { Bridges et al. } \\
2021\end{array}$ \\
\hline AB_Vc_977 & $\Delta n s p S \Delta v c 1807:: \mathrm{Kan}^{\mathrm{R}}$ & $\mathrm{pFY} 4357:: \mathrm{Gm}^{\mathrm{R}}$ & $\mathrm{Sm}, \mathrm{Gm}, \mathrm{Kan}$ & $\begin{array}{l}\text { Bridges et al. } \\
2021\end{array}$ \\
\hline AB_Vc_975 & $\Delta m b a A \Delta v c 1807:: \operatorname{Kan}^{R}$ & pFY4357::Gm & $\mathrm{Sm}, \mathrm{Gm}, \mathrm{Kan}$ & $\begin{array}{l}\text { Bridges et al. } \\
2021\end{array}$ \\
\hline AB_Vc_969 & $\Delta p o t D 1 \Delta v c 1807:: \mathrm{Cm}^{\mathrm{R}}$ & pFY4357::Gm & $\mathrm{Sm}, \mathrm{Gm}, \mathrm{Cm}$ & $\begin{array}{l}\text { Bridges et al. } \\
2021\end{array}$ \\
\hline AB_Vc_1122 & $m b a A-3 x F L A G n s p S-3 x F L A G \quad \Delta v c 1807::$ Spec $^{R}$ & - & Sm, Spec & $\begin{array}{l}\text { NT of } \\
\text { AB_Vc_835 }\end{array}$ \\
\hline AB_Vc_1133 & $m b a A-3 x F L A G n s p S-3 x F L A G \quad \Delta v c 1807::$ Spec $^{R}$ & pFY4357::Gm & Sm, Gm, Spec & $\begin{array}{l}\text { Conj of } \\
\text { AB_Vc_1122 }\end{array}$ \\
\hline AB_Ec_084 & E. coli BL21 (DE3) & $\begin{array}{l}\text { pET15B::npsS- } \\
\text { 6xHis::Amp }{ }^{R}\end{array}$ & Amp & $\begin{array}{l}\text { Transformation } \\
\text { of BL21 }\end{array}$ \\
\hline AB_Vc_835 & $m b a A-3 x F L A G \Delta v c 1807:: \mathrm{Kan}^{\mathrm{R}}$ & - & Sm, Kan & $\begin{array}{l}\text { Bridges et al. } \\
2021\end{array}$ \\
\hline AB_Vc_1182 & $\begin{array}{l}\Delta m b a A \Delta v c 1807:: \text { Pbad-mbaA-3xFLAG::Spec }{ }^{R} \\
\Delta / a c / Z:: \operatorname{Kan}^{R}\end{array}$ & - & Sm, Spec, Kan & $\begin{array}{l}\text { NT of } \\
\text { AB Vc } 1181\end{array}$ \\
\hline JP_Vc_1192 & $\begin{array}{l}\Delta m b a A \Delta v c 1807:: \text { Pbad-mbaA-3xFLAG::Spec } \\
\Delta l a c / Z:: \operatorname{Kan}^{R}\end{array}$ & pFY4357::Gm & Sm, Spec, Kan & $\begin{array}{l}\text { Conj of } \\
A B \_V c \_1182\end{array}$ \\
\hline AB_Vc_960 & $\Delta n s p C \Delta v c 1807:: \operatorname{Kan}^{R}$ & pFY4357::Gm & $\mathrm{Sm}, \mathrm{Gm}, \mathrm{Kan}$ & $\begin{array}{l}\text { Conj of } \\
\text { AB_Vc_823 }\end{array}$ \\
\hline AB_Vc_994 & $\Delta n s p C \Delta$ potD1 $\Delta v c 1807:: \mathrm{Kan}^{\mathrm{R}}$ & $\mathrm{pFY} 4357:: \mathrm{Gm}^{\mathrm{R}}$ & Sm, Gm, Kan & $\begin{array}{l}\text { Conj of } \\
\text { AB_Vc_993 }\end{array}$ \\
\hline AB_Vc_993 & $\Delta n s p C \Delta p o t D 1 \Delta v c 1807:: \mathrm{Kan}^{R}$ & - & Sm, Kan & $\begin{array}{l}\text { NT of } \\
\text { AB_Vc_711 }\end{array}$ \\
\hline AB_Vc_801 & $\Delta v c 1807:: \mathrm{Kan}^{\mathrm{R}}$ & 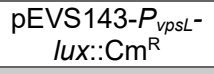 & $\mathrm{Sm}, \mathrm{Cm}, \mathrm{Kan}$ & $\begin{array}{l}\text { Bridges et al. } \\
2020\end{array}$ \\
\hline AB_Vc_1088 & $v p v c^{W 240 R} \Delta v c 1807:: \mathrm{Spec}^{\mathrm{R}}$ & - & Sm, Spec & $\begin{array}{l}\text { NT of } \\
\text { BB Vc } 0090\end{array}$ \\
\hline AB_Vc_839 & $\Delta m b a A \Delta v c 1807:: \mathrm{Kan}^{\mathrm{R}}$ & - & Sm, Kan & $\begin{array}{l}\text { Bridges et al. } \\
2021\end{array}$ \\
\hline AB_Vc_958 & $\Delta v p s L \Delta v c 1807: \mathrm{Kan}^{\mathrm{R}}$ & pFY4357::Gm & Sm, Kan & $\begin{array}{l}\text { Conj of } \\
\text { AB_Vc_487 }\end{array}$ \\
\hline AB_Vc_962 & $\Delta v p s L v p v C^{W 240 R}$ & pFY4357::Gm & $\mathrm{Sm}, \mathrm{Gm}$ & $\begin{array}{l}\text { Conj of } \\
\text { BB_Vc_0101 }\end{array}$ \\
\hline AB_Vc_1145 & Ptac-nspS-mbaA-3xFLAG $\Delta v c 1807:: \mathrm{Spec}^{\mathrm{R}}$ & - & Sm, Spec & $\begin{array}{l}\text { NT of } \\
\text { AB_Vc_835 }\end{array}$ \\
\hline AB_Vc_1203 & $\Delta v p s L$ Ptac-nspS-mbaA-3xFLAG $\Delta v c 1807:: \operatorname{Kan}^{R}$ & pFY4357::Gm & $\mathrm{Sm}, \mathrm{Gm}, \mathrm{Kan}$ & $\begin{array}{l}\text { Conj of } \\
\text { AB_Vc_1197 }\end{array}$ \\
\hline AB_Vc_996 & $\Delta c d g L \Delta v c 1807:: \mathrm{Kan}^{\mathrm{R}}$ & - & Sm, Kan & $\begin{array}{l}\text { NT of } \\
\text { BB_Vc_0090 }\end{array}$ \\
\hline JP_Vc_1206 & $\Delta c d g L \Delta v c 1807:: \operatorname{Kan}^{R}$ & pFY4357::Gm & $\mathrm{Sm}, \mathrm{Gm}, \mathrm{Kan}$ & $\begin{array}{l}\text { Conj of } \\
\text { AB_Vc_996 }\end{array}$ \\
\hline AB_Vc_1056 & $\Delta v c 1807:: P b a d-c d g L:: \operatorname{Kan}^{R}$ & - & Sm, Kan & $\begin{array}{l}\text { NT of } \\
\text { BB_Vc_0090 }\end{array}$ \\
\hline AB_Vc_1104 & $\Delta v c 1807::$ Pbad-cdgL::Kan ${ }^{\mathrm{R}}$ & $\mathrm{pFY} 4357:: \mathrm{Gm}^{\mathrm{R}}$ & $\mathrm{Sm}, \mathrm{Gm}, \mathrm{Kan}$ & Conj of 1056 \\
\hline AB_Vc_1124 & $\Delta v p v C \Delta v c 1807:: \mathrm{Spec}^{R}$ & - & Sm, Spec & $\begin{array}{l}\text { NT of } \\
\text { BB_Vc_0090 }\end{array}$ \\
\hline JP_Vc_1205 & $\Delta v p v C \Delta v c 1807:: \mathrm{Spec}^{\mathrm{R}}$ & pFY4357::Gm & Sm, Gm, Spec & $\begin{array}{l}\text { Conj of } \\
\text { AB_Vc_1124 }\end{array}$ \\
\hline AB_Vc_1109 & $\Delta v c 1807::$ Pbad-vpvC ${ }^{W 240 R:: \mathrm{Kan}^{\mathrm{R}}}$ & - & Sm, Kan & $\begin{array}{l}\text { NT of } \\
\text { BB_Vc_0090 }\end{array}$ \\
\hline AB_Vc_1110 & $\Delta v c 1807::$ Pbad-vpvC ${ }^{W 240 R}:: \mathrm{Kan}^{R}$ & pFY4357::Gm & $\mathrm{Sm}, \mathrm{Gm}, \mathrm{Kan}$ & Conj of 1109 \\
\hline
\end{tabular}

NT $=$ Natural Transformation

Conj $=$ Conjugation 
Table S2. DNA oligonucleotides and gene fragments used in this study.

\begin{tabular}{|c|c|c|c|c|}
\hline $\begin{array}{l}\text { Oligo } \\
\#\end{array}$ & Name & Purpose & Direction & 5 ' to $3^{\prime}$ Sequence \\
\hline 691 & npsS_3000up & $\begin{array}{l}\text { Cloning at } n s p s \\
\quad \text { locus }\end{array}$ & $\mathrm{F}$ & GACTTTATCAGGCCTACTCGCGTTATCCCTG \\
\hline 890 & npsS_3xFlag_B & $\begin{array}{l}\text { Cloning nspS- } \\
\quad 3 x F L A G\end{array}$ & $\mathrm{R}$ & CGCTGCTCTGGGAGATAAATCGAGAGATC \\
\hline 981 & $\begin{array}{l}\text { nspS_3xFLAG_- } \\
\text { GSLinker_Gblock }\end{array}$ & $\begin{array}{l}\text { Cloning nspS- } \\
\quad 3 x F L A G\end{array}$ & $\mathrm{~F}$ & $\begin{array}{c}\text { GATCTCTCGATTTATCTCCCAGAGCAGCGATTGGCAGAAGGTATCA } \\
\text { TTGATTCTGAACTTTCTGCTAAGAATCTGTCTCTTCGTGCTAAGATTA } \\
\text { TCTCTTCAGTTACGTATCAGTACGAAGCGAAACCTTACGGTTCAGGA } \\
\text { AGTGGTAGTGGATCTGACTACAAAGACCATGACGGTGATTATAAAG } \\
\text { ATCATGATATCGATTACAAGGATGACGATGACAAGTGATCAGCAGT } \\
\text { GTGACTTACCAATATGAAGCTAAACCATAG }\end{array}$ \\
\hline 891 & npsS_3xFlag_C & $\begin{array}{l}\text { Cloning nspS- } \\
\quad 3 x F L A G\end{array}$ & $\mathrm{~F}$ & CAGCAGTGTGACTTACCAATATGAAGCTAAACCATAG \\
\hline 698 & npsS_3000dwn & $\begin{array}{l}\text { Cloning at } n s p S \\
\text { locus }\end{array}$ & $\mathrm{R}$ & CGCGTGGTTCGAATGAGCTCAAGTC \\
\hline 692 & npsS_2700up & $\begin{array}{l}\text { Cloning at } n s p S \\
\quad \text { locus }\end{array}$ & $\mathrm{F}$ & CTCTACAAAGCGGAACGTGGTTAACCG \\
\hline 697 & npsS_2700dwn & $\begin{array}{l}\text { Cloning at } n s p S \\
\text { locus }\end{array}$ & $\mathrm{R}$ & ССTAATGACATCACTGGCTAAGCCGAG \\
\hline 862 & pET15B_RBS & $\begin{array}{l}\text { pET15b-nspS- } \\
\quad 6 x H i s\end{array}$ & $\mathrm{R}$ & $\begin{array}{l}\text { CATGGTATATCTCCTTCTTAAAGTTAAACAAAATTATTTCTAGAGGGG } \\
\text { AATTG }\end{array}$ \\
\hline 863 & pET15B_cleav6His & $\begin{array}{l}\text { pET15b-nspS- } \\
\quad 6 x \text { His }\end{array}$ & $\mathrm{F}$ & GGAAGCAGTGGTAGCGGCCTGGTGCC \\
\hline 864 & pET15B_npsS & $\begin{array}{l}\text { pET15b-nspS- } \\
\quad 6 x \text { His }\end{array}$ & $\mathrm{F}$ & $\begin{array}{c}\text { GAAATAATTTTGTTTAACTTTAAGAAGGAGATATACCATGGAACTGAA } \\
\text { TGTCTACCTTTGGGAAGATAC }\end{array}$ \\
\hline 865 & pET15B_npsS & $\begin{array}{l}\text { pET15b-nspS- } \\
\quad 6 \times \text { His }\end{array}$ & $\mathrm{R}$ & $\begin{array}{l}\text { GGCACCAGGCCGCTACCACTGCTTCCTGGTTTAGCTTCATATTGGT } \\
\text { AAGTCACACTG }\end{array}$ \\
\hline 577 & mbaA_100up & $\begin{array}{l}\text { Cloning at } m b a A \\
\text { locus }\end{array}$ & $\mathrm{F}$ & GAAACCTGACATTGCCGCAATCAATGC \\
\hline 893 & 3xFLAG_AbR & $\begin{array}{c}\Delta v c 1807:: \text { Pbad- } \\
m b a A- \\
3 x F L A G:: \text { Spec }^{R}\end{array}$ & $\mathrm{R}$ & $\begin{array}{l}\text { GTCGACGGATCCCCGGAATTCACTTGTCATCGTCATCCTTGTAATC } \\
\text { G }\end{array}$ \\
\hline 986 & vc1807_3xFLAG_E & $\begin{array}{c}\Delta v c 1807:: \text { Pbad- } \\
m b a A- \\
3 x F L A G:: \text { Spec }^{R}\end{array}$ & $\mathrm{~F}$ & $\begin{array}{l}\text { CGATTACAAGGATGACGATGACAAGTGAATTCCGGGGATCCGTCGA } \\
\text { CCTGCAG }\end{array}$ \\
\hline 230 & vc1807_100dwn & $\begin{array}{l}\Delta v c 1807:: \text { Pbad- } \\
\text { mbaA- } \\
\text { 3xFLAG::Spec }{ }^{R}\end{array}$ & $\mathrm{R}$ & GGTAAAGTCGTAGGCTCTGTCGCTG \\
\hline
\end{tabular}


bioRxiv preprint doi: https://doi.org/10.1101/2021.09.21.461265; this version posted September 21,2021 . The copyright holder for this preprint (which was not certified by peer review) is the author/funder, who has granted bioRxiv a license to display the preprint in perpetuity. It is made available under aCC-BY-NC 4.0 International license.

\begin{tabular}{|c|c|c|c|c|}
\hline 1011 & $n s p S \_100 d w n$ & $\begin{array}{c}\Delta v c 1807:: \text { Pbad- } \\
\text { mbaA- } \\
\text { 3xFLAG::Spec }\end{array}$ & $\mathrm{F}$ & CACGCTGGCCAAAAGTGACATCATC \\
\hline 233 & ABD124 & $\begin{array}{c}\Delta v c 1807:: \text { Pbad- } \\
m b a A- \\
3 x F L A G:: \text { Spec }^{R}\end{array}$ & $\mathrm{R}$ & TGTAGGCTGGAGCTGCTTC \\
\hline 571 & mbaA_3000up & $\begin{array}{l}\text { Endogenous } \\
\text { Ptac-nspS-mbaA }\end{array}$ & $\mathrm{F}$ & GCGCGCTAATCTGAACTCAACCCATAAG \\
\hline 1015 & Ptac-nspS_ENDO_B & $\begin{array}{l}\text { Endogenous } \\
\text { Ptac-nspS-mbaA }\end{array}$ & $\mathrm{R}$ & $\begin{array}{l}\text { CTGACGCCAGAAGCATTGGTGCAGATCCGACAAATAACCTAATAGC } \\
\text { GTAAAAG }\end{array}$ \\
\hline 1016 & Ptac-nspS_ENDO_C & $\begin{array}{l}\text { Endogenous } \\
\text { Ptac-nspS-mbaA }\end{array}$ & $\mathrm{F}$ & $\begin{array}{c}\text { CTTTTACGCTATTAGGTTATTTGTCGGATCTGCACCAATGCTTCTGG } \\
\text { CGTCAG }\end{array}$ \\
\hline 971 & nspS_3xFlag_rev & $\begin{array}{l}\text { Endogenous } \\
\text { Ptac-nspS-mbaA }\end{array}$ & $\mathrm{R}$ & CTATGGTTTAGCTTCATATTGGTAAGTCACACTGCTG \\
\hline 572 & mbaA_2700up & $\begin{array}{l}\text { Endogenous } \\
\text { Ptac-nspS-mbaA }\end{array}$ & $\mathrm{F}$ & CGTTAGCATTCCACGCGGTCAGTTAG \\
\hline 890 & npsS_3xFlag_B & $\begin{array}{l}\text { Endogenous } \\
\text { Ptac-nspS-mbaA }\end{array}$ & $\mathrm{R}$ & CGCTGCTCTGGGAGATAAATCGAGAGATC \\
\hline 878 & cdgL_3000up & $\begin{array}{l}\text { Cloning at } c d g L \\
\text { locus }\end{array}$ & $\mathrm{F}$ & CGCCGATTACCTTCAATCTCAAGGTATC \\
\hline 881 & $c d g L_{-} B$ & Deleting $c d g L$ & $\mathrm{R}$ & $\begin{array}{l}\text { CAAATTTCAAATGATGTATCGTTTAAAATATCACCATCGTCATAATAA } \\
\text { ACCTTTAC }\end{array}$ \\
\hline 882 & $c d g L_{-} \mathrm{C}$ & Deleting $c d g L$ & $\mathrm{~F}$ & $\begin{array}{l}\text { GTAAAGGTTTATTATGACGATGGTGATATTTTAAACGATACATCATTT } \\
\text { GAAATTTG }\end{array}$ \\
\hline 885 & cdgL_3000down & $\begin{array}{l}\text { Cloning at } c d g L \\
\text { locus }\end{array}$ & $\mathrm{R}$ & GTGAGTTTCGCTCGAACCTGCATCG \\
\hline 879 & cdgL_2700up & $\begin{array}{l}\text { Cloning at } c d g L \\
\text { locus }\end{array}$ & $\mathrm{F}$ & GTTCGTTACTCGCATATCATCGATCCAACTAC \\
\hline 884 & cdgL_2700down & $\begin{array}{l}\text { Cloning at } c d g L \\
\text { locus }\end{array}$ & $\mathrm{R}$ & GTAGCGGGTGTAGATCTGCTGCGTTC \\
\hline 105 & BBC1881 & $\begin{array}{l}\Delta v c 1807:: \text { Pbad- } \\
\quad \text { cloning }\end{array}$ & $\mathrm{F}$ & TTTAAAGGGGATCAGTGACCG \\
\hline 721 & $\begin{array}{l}\text { Pbad_vc1807_- } \\
\text { Universal_B }\end{array}$ & $\begin{array}{l}\Delta v c 1807:: P b a d- \\
\quad \text { cloning }\end{array}$ & $\mathrm{R}$ & САTTTCACACСTCСTGCAGGTAC \\
\hline 911 & Pbad_cdgL_C & $\begin{array}{l}\Delta v c 1807:: P_{\text {Pbad- }} \\
\quad c d g L:: \mathrm{Kan}^{\mathrm{R}}\end{array}$ & $\mathrm{F}$ & $\begin{array}{l}\text { GTACCTGCAGGAGGTGTGAAATGAATTTAAATAACTTTAGCCTACGC } \\
\text { TGGCTAAC }\end{array}$ \\
\hline
\end{tabular}


bioRxiv preprint doi: https://doi.org/10.1101/2021.09.21.461265; this version posted September 21, 2021. The copyright holder for this preprint (which was not certified by peer review) is the author/funder, who has granted bioRxiv a license to display the preprint in perpetuity. It is made available under aCC-BY-NC 4.0 International license.

\begin{tabular}{|c|c|c|c|c|}
\hline 912 & Pbad_cdgL_D & $\begin{array}{l}\Delta v c 1807:: \text { Pbad- } \\
\quad c d g L:: \operatorname{Kan}^{R}\end{array}$ & $\mathrm{R}$ & $\begin{array}{l}\text { GTCGACGGATCCCCGGAATTTAGACAAAATTTCGCACAACGTATCG } \\
\text { ATC }\end{array}$ \\
\hline 232 & ABD123 & $\begin{array}{l}\Delta v c 1807:: P b a d- \\
\quad \text { cloning }\end{array}$ & $\mathrm{F}$ & ATTCCGGGGATCCGTCGAC \\
\hline 106 & BBC1882 & $\begin{array}{l}\Delta v c 1807:: \text { Pbad- } \\
\quad \text { cloning }\end{array}$ & $\mathrm{R}$ & CAATTTTGCTTTTGGACCATCCC \\
\hline 978 & Pbad_vpvC_fwd & $\begin{array}{l}\Delta v c 1807:: P b a d- \\
v p v C^{W 240 R:: K_{\text {Kan }}^{R}}\end{array}$ & $\mathrm{~F}$ & GTACCTGCAGGAGGTGTGAAATGACTGATCAAACGCGAACTTCGC \\
\hline 979 & Pbad_vpvC_rev & $\begin{array}{l}\Delta v c 1807:: \text { Pbad- } \\
\text { vpvC }{ }^{W 240 R}:: \text { Kan }^{R}\end{array}$ & $\mathrm{R}$ & $\begin{array}{l}\text { GTCGACGGATCCCCGGAATCTATCTGAACTGATCCTGCTTGAGTTC } \\
\text { TTTCGC }\end{array}$ \\
\hline 203 & vpvC_3000up & $\begin{array}{l}\text { Cloning at } v p v C \\
\text { locus }\end{array}$ & $\mathrm{F}$ & CGATCCAGTGCATGCAGCGGAAATTGC \\
\hline 998 & $v p v C \_B$ & Deleting $v p v C$ & $\mathrm{R}$ & $\begin{array}{l}\text { GATGGCGAAAGTGCTCGAAGCCTCTCGCGAAGAGCACCAGTAATAC } \\
\text { TTTCAGCGCG }\end{array}$ \\
\hline 999 & vpvC_C & Deleting $v p v C$ & $\mathrm{~F}$ & $\begin{array}{c}\text { CGCGCTGAAAGTATTACTGGTGCTCTTCGCGAGAGGCTTCGAGCAC } \\
\text { TTTCGCCATC }\end{array}$ \\
\hline 204 & vpvC_3000down & $\begin{array}{l}\text { Cloning at } v p v C \\
\text { locus }\end{array}$ & $\mathrm{R}$ & CCGGCTGATGCTTTGTGTCTAACGTGCTG \\
\hline 997 & vpvC_2700up & $\begin{array}{l}\text { Cloning at } v p v C \\
\text { locus }\end{array}$ & $\mathrm{F}$ & СTCACTGCGGCTGGCATTAAAGTTTCTCTG \\
\hline 1001 & vpvC_2700down & $\begin{array}{l}\text { Cloning at } v p v C \\
\text { locus }\end{array}$ & $\mathrm{R}$ & GCTCACTTTGGCACCTACCCTGATTATTG \\
\hline
\end{tabular}

